ArXiv:1010.2720

AEI-2010-156

LPT ENS-10/47

Commun. Math. Phys. 311, 787-814 (2012)

Digital Object Identier (DOI) 10.1007/s00220-012-1428-9

\title{
Baxter's Q-operators and operatorial Bäcklund flow for quantum (super)-spin chains
}

\author{
Vladimir Kazakov ${ }^{1,2, \star}$, Sebastien Leurent ${ }^{1}$, and Zengo Tsuboi ${ }^{3,4, \star \star}$ \\ 1 Ecole Normale Superieure, LPT, 75231 Paris CEDEX-5, France \\ 2 Université Paris-VI, Paris, France \\ 3 Max-Planck-Institut für Gravitationsphysik, Albert-Einstein-Institut, Am Mühlenberg 1, \\ 14476 Potsdam, Germany \\ 4 Osaka City University Advanced Mathematical Institute, 3-3-138 Sugimoto, Sumiyoshi-ku \\ Osaka 558-8585 Japan
}

\begin{abstract}
We propose the operatorial Baxter's TQ-relations in a general form of the operatorial Bäcklund flow describing the nesting process for the inhomogeneous rational $g l(K \mid M)$ quantum (super)spin chains with twisted periodic boundary conditions. The full set of Q-operators and T-operators on all levels of nesting is explicitly defined. The results are based on a generalization of the identities among the group characters and their group co-derivatives with respect to the twist matrix, found by one of the authors and P.Vieira 11. Our formalism, based on this new "master" identity, allows a systematic and rather straightforward derivation of the whole set of nested Bethe ansatz equations for the spectrum of quantum integrable spin chains, starting from the R-matrix.
\end{abstract}

\footnotetext{
* member of Institut Universitaire de France

$\star \star$ Present address: Institut für Mathematik und Institut für Physik, Humboldt-Universität zu Berlin, Johann von Neumann-Haus, Rudower Chaussee 25, 12489 Berlin, Germany
} 


\section{Introduction}

It has been noticed long ago that the mathematical structures behind the quantum integrable spin chains have many similarities with the theory of classical integrable systems, such as KP or KdV hierarchies. It goes of course not only about the obvious correspondence between the quantum integrable systems and their classical limits when, for example, the quantum transfer matrix of a quantum 1+1-dimensional system becomes the classical monodromy matrix of the corresponding classical Lax connection. There is a more striking "classical" feature of the quantum integrability: The quantum transfer matrix represents a natural (spectral parameter dependent) generalization of the Schur character of a classical algebra [2 given by the so called Bazhanov-Reshetikhin (BR) determinant formula and, as such, it satisfies a certain Hirota bilinear finite difference equation, which appears in the quantum context as a certain fusion relation among the composite quantum states appearing in quantum spin chains as certain bound states ("strings") of Bethe roots [3, 4,5,6,7. Similar, though a more complicated realization of Hirota discrete "classical" integrable dynamics has been observed in the context of the quantum $(1+1)$-dimensional QFT's, or sigma-models 6, 8, 9, an observation which appeared to be at the heart of an important advance in the study of the spectrum of the $\mathrm{AdS}_{5} / \mathrm{CFT}_{4}$ correspondence 10, 11.

Hirota equation immediately brings us to the idea that quantum integrability, at least for certain quantities, such as transfer-matrix eigenvalues, can be viewed as a specific case of classical integrability and of the theory of classical tau-functions. Indeed, the character of a classical group, say $g l(K)$, is nothing but a tau-function of the $\mathrm{KdV}$ hierarchy. It was proposed in [1] to view the quantum transfer matrix of a rational quantum Heisenberg-type $g l(K \mid M)$ (super)-spin chain with twisted boundary conditions as a quantum, operatorial generalization of the character and to construct the transfer matrix (T-operator) by acting on the character in a given irrep, as a function of the group element (twist), by special group derivatives, called the co-derivatives. The formalism of co-derivatives has led to a direct proof of the BR formula [1] (see also [12]), and the basic underlying identity for the characters found in [1] seems to be just a new form of the KdV Hirota identity (the fact yet to be understood).

In the present paper, we want to move even further in this classical interpretation of the quantum integrability and to generalize the basic identity of [1] to include the Baxter's TQ-relations into our formalism. This implies a natural definition of all Baxter's Q-operators, rather different from the one known in the literature $13,6,14,15,16,17,18,19$, and more generally, of the T-operators on all levels of the nesting procedure. This nesting takes a form of a Bäcklund flow, directly for the T- and Q-operators. Due to the fact that all of them belong to a commuting family of operators, all these relations can be immediately transformed into the well known functional form, for their eigenvalues 20,21 , 22 .23.

Our main identity given in the next section offers an interesting alternative and a concise approach to the quantum integrability uncovering the whole structure of the nested Bethe ansatz, from the R-matrix and the Yang-Baxter relations all the way to the nested Bethe ansatz equations, in the general operatorial form for all the intermediate quantities. 


\section{Transfer-matrix, co-derivative and TQ-relations}

We recall that the main object of our study is the transfer matrix of an inhomogeneous quantum spin chain 1

$$
\mathbf{T}^{\{\lambda\}}(u)=\operatorname{tr}_{\lambda}\left(\mathbf{R}_{N}^{\{\lambda\}}\left(u-\theta_{N}\right) \otimes \cdots \otimes \mathbf{R}_{1}^{\{\lambda\}}\left(u-\theta_{1}\right) \pi_{\lambda}(g)\right)
$$

where $\pi_{\lambda}(g)$ is a matrix element of a twist matrix $g \in G L(K)$ in an irrep $\lambda$ and

$$
\mathbf{R}_{i}^{\{\lambda\}}(u)=u \mathbb{I}+2 \sum_{\alpha \beta} e_{\beta \alpha}^{(i)} \otimes \pi_{\lambda}\left(e_{\alpha \beta}\right) \equiv u+2 \mathcal{P}_{i, \lambda}
$$

is the R-matrix in irrep $\lambda$ in auxiliary space (and in fundamental irrep in the quantum space). The identity operator $\mathbb{I}$ is implicit in the r.h.s., it will often be omitted for the brevity. The $g l(K)$ generator $e_{\alpha \beta}^{(i)}$ corresponds to the $i^{\text {th }}$ quantum space 2 (which is in the fundamental representation) and $\pi_{\lambda}\left(e_{\alpha \beta}\right)$ to the auxiliary space. When $\lambda$ is also fundamental then $\mathcal{P}_{i, \lambda}$ becomes a usual permutation operator $e_{\beta \alpha}^{(i)}=\mathbb{I}^{\otimes(i-1)} \otimes e_{\beta \alpha} \otimes \mathbb{I}^{\otimes(N-i)}$ so that $\mathcal{P}_{i}=\sum_{\alpha \beta} e_{\beta \alpha}^{(i)} \otimes e_{\alpha \beta}$. $\mathcal{P}_{i}$ permutes the indices of the auxiliary space and the quantum subspace. This $\mathbf{T}^{\{\lambda\}}(u)$ is a spectral parameter $u \in \mathbb{C}$ dependent operator on the quantum space $\mathcal{H}$. It is polynomial in $u$ and in the inhomogeneities $\theta_{i} \in \mathbb{C}$.

The main goal is to find all the eigenvalues of this transfer matrix for the $g l(K)$ quantum spins. For that we work out an operatorial Bäcklund transformation, which can be also called the nesting procedure, whose main goal is to derive, in a deductive way, without any assumptions, the nested system of Bethe ansatz equations defining these eigenvalues. On the way, we will encounter a collection of the intermediate T-operators, and the Baxter's Q-operators as their particular case, at each level of nesting. The operatorial TQ-relations, representing the Bäcklund transformation reducing the problem for a $g l(k)$ subalgebra to a similar problem for the $g l(k-1)$ subalgebra, in the nesting procedure corresponding to the chain of embeddings $g l(K) \supset g l(K-1) \supset \cdots \supset g l(1)$ were given in their functional form in [21] (for the super-spin chains in [20]). All the T- and Qoperators in this nesting procedure are labelled by the subsets $I$ of the full set $\mathfrak{I}=\{1,2, \ldots, K\}$ as $\mathbf{T}_{I}^{\{\lambda\}}(u)$ and $\mathbf{Q}_{I}(u)$. The original transfer matrix (2) corresponds to the T-operator for the full set $\mathbf{T}^{\{\lambda\}}(u)=\mathbf{T}_{\mathfrak{I}}^{\{\lambda\}}(u)$. There are $2^{K}$ subsets of the full set $\mathfrak{I}$ and they can be described in terms of the Hasse diagram based on the inclusion relations. A chain of subsets of the full set $\mathfrak{I}=I_{K} \supset$ $I_{K-1} \supset \cdots \supset I_{0}=\emptyset$, where $\left|I_{k}\right| \equiv \operatorname{Card}\left(I_{k}\right)=k$, forms a path on the Hasse diagram. We will call this the nesting path, and $(K-k)$ - the level of nesting. There are $K$ ! different nesting paths for $g l(K)$ (see fig 1b). This description of

1 Throughout this paper, all operators will act on the same Hilbert "quantum" space $\mathcal{H}=\left(\mathbb{C}^{K}\right)^{\otimes N}\left(\operatorname{resp}\left(\mathbb{C}^{K \mid M}\right)^{\otimes N}\right.$ for supersymmetric spin chains). We use the Young diagram $\lambda$ to label the irreducible tensor representation. The fundamental representation corresponds to the Young diagram with one box.

2 Here $\left\{e_{i j}\right\}_{i, j=1}^{K}$ satisfy the commutation relations $\left[e_{i j}, e_{k l}\right]=\delta_{j k} e_{i l}-\delta_{l i} e_{k j}$. The $i^{\text {th }}$ quantum space is the $i^{\text {th }}$ factor in $\mathcal{H}=\underbrace{\left(\mathbb{C}^{K}\right) \otimes\left(\mathbb{C}^{K}\right) \otimes \cdots \otimes\left(\mathbb{C}^{K}\right)}_{N \text { times }}$. In this way, e.g. $e_{\beta \alpha}^{(i)}=$ $\mathbb{I}^{\otimes(i-1)} \otimes e_{\beta \alpha} \otimes \mathbb{I}^{\otimes(N-i)}$. The generalization of our construction to the case of $g l(K \mid M)$ superspins will be given in sec 5 
all the $2^{K}$ Q-operators based on the Hasse diagram was proposed in 23, and will be used throughout this paper.

The general TQ-relations, derived in sec 4 in the operatorial form, are given by

$$
\mathbf{T}_{I}^{s}(u) \mathbf{Q}_{I, j}(u)=\mathbf{T}_{I, j}^{s}(u) \mathbf{Q}_{I}(u)-x_{j} \mathbf{T}_{I, j}^{s-1}(u+2) \mathbf{Q}_{I}(u-2),
$$

where $g=\operatorname{diag}\left(x_{1}, x_{2}, \cdots, x_{K}\right)$ is the twist matrix in the diagonal basis, the superscript $s$ in T-operator denotes the symmetric $\lambda=(s)$ irrep in the auxiliary space, by $I \subset \mathfrak{I}=\{1,2, \cdots, K\}$ we denote a subset of the full set of indices (labeling the eigenvalues) and by $I, j \equiv I \cup\{j\} \subset \mathfrak{I}$ we denote a subset with one more index $j \notin I$. This TQ-relation relates the T-operator $\mathbf{T}_{I}^{s}$ and the Toperator $\mathbf{T}_{I, j}^{s}$ of the previous level of nesting (which has one more index). A chain of these relations allows to relate the original transfer matrix $\mathbf{T}_{\mathfrak{I}}^{s}(u)$ on the level zero of nesting to the $u$-independent operator $\mathbf{T}_{\emptyset}^{s}(u)$ given by (41). We will also show that the Q-operators are equal to the T-operators taken at an empty Young diagram:

$$
\mathbf{Q}_{I}(u)=\mathbf{T}_{I}^{0}(u) .
$$

In the papers 21,20 , all the $\mathrm{T}$ - and Q-operators at intermediate steps were assumed, by self-consistency and without a proof, to be polynomials in $u$. This analyticity assumption immediately leads to the nested Bethe ansatz equations defined by the nesting path. In this paper, we complete the missing link of the chain and find the explicit operatorial form of the Bäcklund flow (3).

In what follows, we will extensively use the definitions and the identities of 1. In particular, the co-derivative $\hat{D}$ defined there and used through the whole current paper is a very simple object defined by its action on any function of $g$ as follows

$$
\hat{D} \otimes f(g)=\left.\frac{\partial}{\partial \phi} \otimes f\left(e^{\phi \cdot e} g\right)\right|_{\phi=0}
$$

where $\phi$ is a matrix in the fundamental representation: $\phi \cdot e \equiv \sum_{\alpha \beta} e_{\alpha \beta} \phi_{\beta}^{\alpha}$ and $\frac{\partial}{\partial \phi}=\sum_{\alpha \beta} e_{\alpha \beta} \frac{\partial}{\partial \phi_{\alpha}^{\beta}}$. Its main property, which also could serve as its definition, manifests in its action on the group element in fundamental irrep:

$$
\hat{D} \otimes g=\mathcal{P}(1 \otimes g)
$$

where $\mathcal{P}$ is the operator of permutation between the $1^{\text {st }}$ and the $2^{\text {nd }}$ spaces 3 .

Many other useful properties of this co-derivative, mostly following from the application of the standard Leibniz rule can be found in [1] and some of them are summarized in the appendix A.1

Using the co-derivative we can for example rewrite the T-operator (1) in the following way:

$$
\mathbf{T}^{\{\lambda\}}(u)=\left(u_{1}+2 \hat{D}\right) \otimes\left(u_{2}+2 \hat{D}\right) \otimes \cdots \otimes\left(u_{N}+2 \hat{D}\right) \chi_{\{\lambda\}}(g)
$$

3 Explicitly in indices, the last relation looks like $\hat{D}_{j_{1}}^{i_{1}} g_{j_{2}}^{i_{2}}=\delta_{j_{1}}^{i_{2}} g_{j_{2}}^{i_{1}}$. It is a usual matrix derivative obeying the Leibniz rules. Throughout this paper, we define the tensor (or matrix) indices of any operator $\mathbf{A}$ with respect to a basis $\left\{v_{k_{1}} \otimes v_{k_{2}} \otimes \cdots \otimes v_{k_{N}}\right\}$ as $\mathbf{A} v_{l_{1}} \otimes v_{l_{2}} \otimes \cdots \otimes v_{l_{N}}=$ $\sum_{k_{1}, k_{2}, \cdots, k_{N}} A_{l_{1}, l_{2}, \cdots, l_{N}}^{k_{1}, k_{2}, \cdots, k_{N}} v_{k_{1}} \otimes v_{k_{2}} \otimes \cdots \otimes v_{k_{N}}$. In particular, a usual notation of a matrix element $A_{k, l}$ is written as $A_{l}^{k}$. 


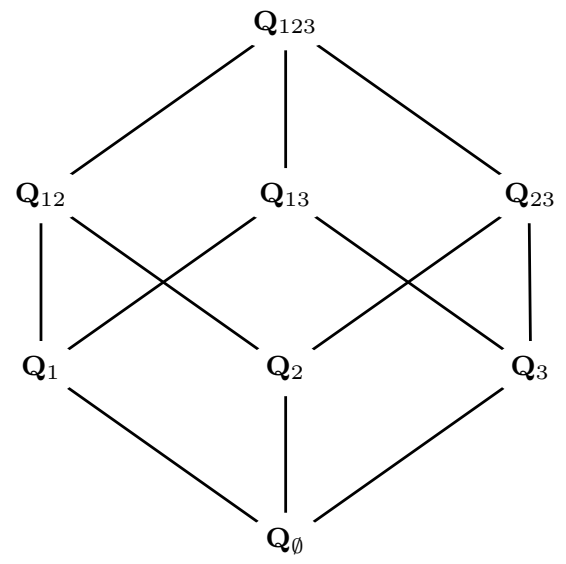

(a) Hasse diagram for the Q-operators: $g l(3)$ case. There are $2^{3}=8$ Q-operators.

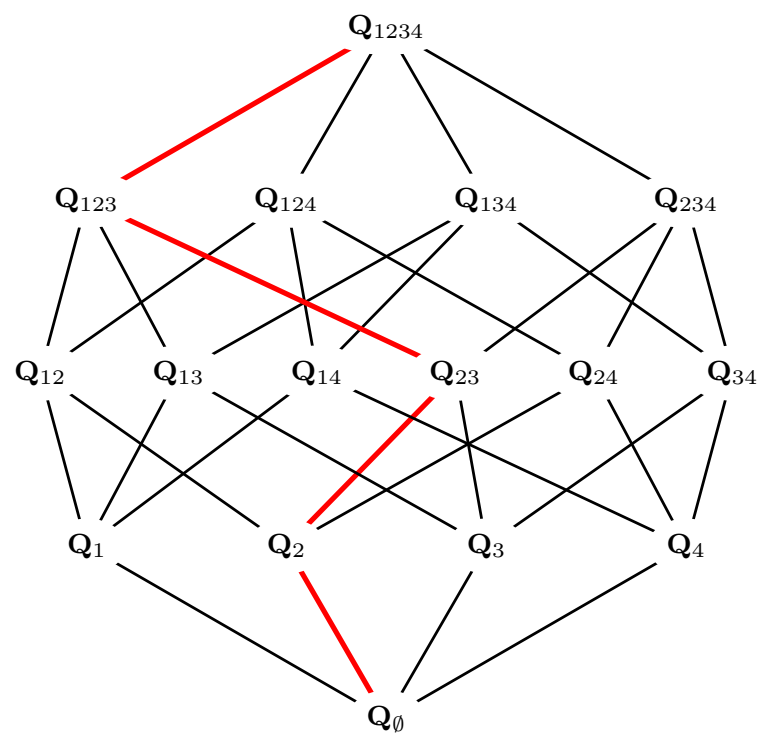

(b) Hasse diagram for the Q-operators: $g l(4)$ case. There are $2^{4}=16$ Q-operators.

Fig. 1: Hasse diagram for the Q-operators in $g l(3)$ and $g l(4)$ case. In (b), the red thick lines denote one of the $4 !=24$ nesting paths characterized by a chain of the index sets $\mathfrak{I}=I_{4} \supset I_{3} \supset I_{2} \supset I_{1} \supset I_{0}$, where $I_{4}=\{1,2,3,4\}, I_{3}=$ $\{1,2,3\}, I_{2}=\{2,3\}, I_{1}=\{2\}, I_{0}=\emptyset$. Note that (b) contains the diagram (a) for $g l(3)$ as a subdiagram. 
where $u_{i}=u-\theta_{i}$ and $\chi_{\{\lambda\}}(g)=\operatorname{tr} \pi_{\lambda}(g)$ is the character of the twist $g$ in the irrep $\lambda$. The action of each of $N$ brackets adds a new spin to the system, with its fundamental quantum space.

\section{The master identity}

In this section we will formulate the main identity of this paper, called here the master identity (8) - the basis of our approach to the quantum integrability. It is proven in the appendix $\mathrm{E}$.

This identity involves the generating function of characters in symmetric $(\lambda=$ $(s))$ or antisymmetric $\left(\lambda=\left(1^{a}\right)\right)$ tensor irreps,

$$
w(z)=\operatorname{det} \frac{1}{1-z g}=\frac{1}{\prod_{j=1}^{K}\left(1-z x_{j}\right)}=\sum_{s=0}^{\infty} z^{s} \chi_{s}(g)=\frac{1}{\sum_{a=0}^{\infty}(-1)^{a} z^{a} \chi^{(a)}(g)},
$$

where $x_{j}$ are the eigenvalues 4 of the twist matrix $g$, and $z \in \mathbb{C}$. This master identity will relate operators of the form $\otimes_{i}\left(u_{i}+2 \hat{D}\right) \prod_{k} w\left(t_{k}\right)$ (for an arbitrary set of complex numbers $\left\{t_{k}\right\}$ ), which is a generalization 5 of the T-operator (6). To avoid the bulky notations in this definition, we assume all the terms like $u_{i}$ and $2+u_{i}$ to be multiplied by the identity operator $\mathbb{I}$, and the tensor product $\otimes_{i}$ is taken a: $\vec{\otimes}_{i=1}^{N}$ unless it is explicitly stated otherwise. Due to the commutation relation (71) following from the Yang-Baxter relation, the operators $\otimes_{i}\left(u_{i}+\right.$ $2 \hat{D}) \prod_{k} w\left(t_{k}\right)$ are conserved charges, in the sense that they belong to the same family of commuting operators as the T-operators.

The master identity reads as follows $(t, z \in \mathbb{C})$ :

$$
\begin{aligned}
&(t-z)\left[\otimes_{i}\left(2+u_{i}+2 \hat{D}\right) w(z) w(t) \Pi\right] \cdot\left[\otimes_{i}\left(u_{i}+2 \hat{D}\right) \Pi\right] \\
&=t\left[\otimes_{i}\left(u_{i}+2 \hat{D}\right) w(z) \Pi\right] \cdot\left[\otimes_{i}\left(2+u_{i}+2 \hat{D}\right) w(t) \Pi\right] \\
& \quad-z\left[\otimes_{i}\left(2+u_{i}+2 \hat{D}\right) w(z) \Pi\right] \cdot\left[\otimes_{i}\left(u_{i}+2 \hat{D}\right) w(t) \Pi\right]
\end{aligned}
$$

and it holds for any function $\Pi(g)$ of the form $\Pi(g)=\prod_{k} w\left(t_{k}\right)$, or equivalently, $\Pi(g)=\operatorname{det}(f(g))=\prod_{j=1}^{K} f\left(x_{j}\right)$ where $f(z)$ is an arbitrary fixed function analytic in the vicinity of $z=0$. In (8), the dots between consecutive brackets stand for multiplication of operators acting on the quantum space, and each co-derivative operator $\hat{D}$ acts on what lies to its right inside the square brackets.

The proof of our main identity (8) is given in Appendix [E but it can be easily proved directly, for a few small $N$ 's, on Mathematica. The identity represents a natural generalization 7 of the equation (4.1) [equation (20) in the arXiv version] in 1 .

\footnotetext{
4 Throughout this paper, we shall assume that $\forall i \neq j, x_{i} \neq x_{j}$.

5 The T-operator in symmetric irrep can be indeed obtained as $\mathbf{T}^{s}(u)=$ $\frac{1}{s !}\left(\frac{\partial}{\partial z}\right)^{s}\left[\otimes_{i}\left(u_{i}+2 \hat{D}\right) w(z)\right]_{z \rightarrow 0}$.

$6 \vec{\otimes}_{i=1}^{N} A_{i}=A_{1} \otimes A_{2} \otimes \cdots \otimes A_{N}$ for any indexed operators $\left\{A_{i}\right\}_{i=1}^{N}$.

7 The identity (4.1) of [1] corresponds to a particular case when $\Pi=1$ and $\forall i, u_{i}=0$.
} 
To conclude this section, let us demonstrate the use of the master identity (8) by deducing from it a particular case of the Hirota relations for the transfer matrices in particular representations. For that purpose, let us focus on the case when $\Pi=1$. Then, by expending w's in symmetric characters according to (7) and keeping the coefficient of $t^{s} z^{s^{\prime}}$ in each term of (8), we get the relation

$$
\begin{aligned}
{\left[\otimes_{i}\left(2+u_{i}+2 \hat{D}\right)\left(\chi_{s-1} \chi_{s^{\prime}}-\chi_{s} \chi_{s^{\prime}-1}\right)\right] \cdot\left[\prod_{i}\left(u_{i}\right)\right] } \\
=\left[\otimes_{i}\left(u_{i}+2 \hat{D}\right) \chi_{s^{\prime}}\right] \cdot\left[\otimes_{i}\left(2+u_{i}+2 \hat{D}\right) \chi_{s-1}\right] \\
-\left[\otimes_{i}\left(2+u_{i}+2 \hat{D}\right) \chi_{s^{\prime}-1}\right] \cdot\left[\otimes_{i}\left(u_{i}+2 \hat{D}\right) \chi_{s}\right]
\end{aligned}
$$

Choosing $s^{\prime}=s+1$, and rewriting (9) using 8 (6), we get a relation in terms of the T-operators of rectangular Young diagrams :

$$
-\mathbf{T}^{(2, s)}(u+2) \mathbf{T}^{(0, s)}(u)=\mathbf{T}^{(1, s+1)}(u) \mathbf{T}^{(1, s-1)}(u+2)-\mathbf{T}^{(1, s)}(u+2) \mathbf{T}^{(1, s)}(u)
$$

where $(a, s)$ denotes the representation with an $a \times s$ rectangular Young diagram $\lambda=\left(s^{a}\right)$, i.e. $\mathbf{T}^{(a, s)}(u)=\mathbf{T}^{\left\{\left(s^{a}\right)\right\}}(u)$.

Eq. (10), is a particular case of general Hirota relations (35) for the fusion in rational spin chains known since long and proven in [1] at zero level of nesting $(I=\mathfrak{I})$.

\section{Baxter relations for T- and Q-operators}

In this section we will derive from our main identity the operatorial Bäcklund flow in the form of the TQ-relations described above, and even more generally, of TT-relations at every step of the nesting procedure, as well as the QQ-relations 24, 16, 25, 17, 20, 22, 26, 18, (see also earlier papers [27, and a recent presentation in 23. used in this paper) which give an immediate access to the full set of nested Bethe ansatz equations (also written in an operatorial form in quantum space in the subsection 4.5). At the same time, it will give a natural operatorial definition of these quantities on every step of the nesting, and in particular of all the $2^{K}$ Q-operators. Since all these T- and Q-operators belong to the same family of mutually commuting operators, we can transform these relations, at any stage of the nesting procedure, to the operatorial ones, for T- and Q-operators.

4.1. First level of nesting. Now we will obtain from the master identity (8) the operatorial Baxter's TQ-relations. We will start from the first level of nesting. In what follows we will frequently use the notation $\bar{I}=\mathfrak{I} \backslash I$ for the complimentary set of $I$. In particular for any element $j \in \mathfrak{I}$, we use a notation $\bar{j}=\mathfrak{I} \backslash\{j\}$.

\footnotetext{
8 We also use the fact that the characters for rectangular representations $\chi^{(a, s)} \equiv \chi^{\left\{\left(s^{a}\right)\right\}}$, satisfy a simple Hirota relation $\chi^{(a, s+1)} \chi^{(a, s-1)}-\chi^{(a, s)} \chi^{(a, s)}=-\chi^{(a-1, s)} \chi^{(a+1, s)}$.
} 
Definition of Q-operators.

In accord with (4) and (6) (where $\mathbf{T}^{\{\lambda\}}(u)$ denotes $\mathbf{T}_{\bar{\emptyset}}^{\{\lambda\}}(u)$ ), the Q-operator on the zero level of nesting is, by definition,

$$
\mathbf{Q}_{12 \ldots K}(u) \equiv \mathbf{Q}_{\bar{\emptyset}}(u)=\left(\prod_{i=1}^{N} u_{i}\right) \mathbb{I}^{\otimes N}
$$

which is a simple function of $u_{i}$ 's, times the identity operator in the full quantum space. In particular, the last factor in the l.h.s. of (8) becomes $\mathbf{Q}_{\bar{\emptyset}}(u)$ when $\Pi=1$.

We will see in what follows that the Q-operators of the first level of nesting $\mathbf{Q}_{\bar{j}}(u)$ can be defined through the residues at the poles in the expression:

$$
(1-g t)^{\otimes N} \cdot\left[\bigotimes_{i}\left(u_{i}+2 \hat{D}+2\right) w(t)\right]=\sum_{j=1}^{K} \frac{\mathbf{Q}_{\bar{j}}(u)}{1-x_{j} t}+\text { polynomial in } t
$$

where the normalization factor $(1-g t)^{\otimes N}$ is necessary in order to have only simple poles. Indeed, the co-derivative acting on $w(t)$, having simple poles at each $t=x_{j}^{-1}, j=1,2, \ldots, K$, produces the double poles in the same points, as it is clearly seen from (64) in the diagonal basis. The factor $(1-g t)^{\otimes N}$ transforms them again into simple poles, thus justifying the pole expansion in the r.h.s. of (12).

The equivalent definition of the Q-operators is

$$
\mathbf{Q}_{\bar{\jmath}}(u)=\lim _{t \rightarrow \frac{1}{x_{j}}}\left(1-x_{j} t\right)(1-g t)^{\otimes N} \cdot\left[\bigotimes_{i}\left(2+u_{i}+2 \hat{D}\right) w(t)\right] .
$$

This Q-operator acts on the same quantum space $\mathcal{H}=\left(\mathbb{C}^{K}\right)^{\otimes N}$ as the T-operator (6). It is also important to notice that the Q-operator $\mathbf{Q}_{\bar{\jmath}}(u)$ looses its dependence on some $u_{i}$ 's (see appendix $\mathrm{B}$ ). For instance, for one spin $(N=1)$, if we denote9 by $\left|e_{k}\right\rangle$ a basis of eigenstates of $g$, we get

$$
\mathbf{Q}_{\bar{\jmath}}(u)\left|e_{k}\right\rangle=\left\{\begin{aligned}
\left(\left(u_{1}+2\right)\left(1-x_{k} / x_{j}\right)+2 x_{k} / x_{j}\right) \prod_{l \in \bar{\jmath}} \frac{1}{1-x_{l} / x_{j}}\left|e_{k}\right\rangle & \text { if } k \neq j \\
2 \prod_{l \in \bar{\jmath}} \frac{1}{1-x_{l} / x_{j}}\left|e_{k}\right\rangle & \text { if } k=j,
\end{aligned}\right.
$$

where we see that on the state $\left|e_{j}\right\rangle, \mathbf{Q}_{\bar{j}}(u)$ is $u$-independent, while the action of other states is less trivial.

\section{T-operators and TQ-relations.}

Now we will transform the master identity (8) into a set of TQ-relations (3) on the first level of nesting 10 . For that we simply put $\Pi=1$. Multiplying (8) by

\footnotetext{
9 In this definition of the "diagonal basis", $\left|e_{k}\right\rangle$ is such that $g\left|e_{k}\right\rangle=x_{k}\left|e_{k}\right\rangle$.

10 The "first level of nesting" means that we will relate the original T- and Q-operators labeled by the full set $\bar{\emptyset}=\mathfrak{I}$ with some $\mathrm{T}$ and Q-operators labeled by $\bar{j}$, which has one index less.
} 
the matrix $(1-g t)^{\otimes N}$ which commutes with all the factors of both the L.H.S. and the R.H.S 11 , and picking the poles at $t=1 / x_{j}$ we come to the equation

$$
\begin{aligned}
\lim _{t \rightarrow \frac{1}{x_{j}}}\left(1-x_{j} t\right)(1-z / t)(1-g t)^{\otimes N} \cdot\left[\otimes_{i}\left(2+u_{i}+2 \hat{D}\right) w(z) w(t)\right] \cdot \mathbf{Q}_{\bar{\emptyset}}(u)= \\
\quad=\left[\otimes_{i}\left(u_{i}+2 \hat{D}\right) w(z)\right] \cdot \mathbf{Q}_{\bar{j}}(u)-x_{j}\left[\otimes_{i}\left(2+u_{i}+2 \hat{D}\right) z w(z)\right] \cdot \mathbf{Q}_{\bar{j}}(u-2) .
\end{aligned}
$$

It is useful to note that the factor $(1-z / t) \sim\left(1-z x_{j}\right)$ in the L.H.S. can be carried over to the right of the co-derivatives $\hat{D}$ allowing to use the relation

$$
\left(1-z x_{j}\right) w(z)=\left(1-z x_{j}\right) \operatorname{det} \frac{1}{1-z g}=\operatorname{det} \frac{1}{1-z g_{\bar{j}}},
$$

where $g_{\bar{\jmath}}=\operatorname{diag}\left(x_{1}, x_{2}, \cdots, x_{j-1}, x_{j+1}, \cdots, x_{K}\right)$ in the diagonal basis. The possibility to move this factor across the derivatives comes from the factor $(1-g t)^{\otimes N}$, introduced to avoid poles of higher orders in (12). Indeed, for example in the simplest, one spin case $N=1$, we can easily check that $\left(x_{j} \mathbb{I}-g\right) \cdot \hat{D} x_{j}=$ $\left(x_{j} \mathbb{I}-g\right) P_{j} \cdot x_{j}=x_{j}^{2} P_{j}-g P_{j} x_{j}=0$, where $P_{j}$ is the projector on the $j^{\text {th }}$ eigenspace of $g$. The generalization to any $N$ is rather trivial and is discussed in the Appendix $\mathrm{F}$.

Now we introduce the characters of the first level of nesting $\chi_{s}\left(g_{\bar{j}}\right)=\chi_{\{\lambda=(s)\}}\left(g_{\bar{j}}\right)$ corresponding to the symmetric tensor representations of the sub-algebra $g l(K-1) \subset g l(K)$, defined by the generating function

$$
w_{\bar{j}}(z) \equiv \operatorname{det} \frac{1}{1-z g_{\bar{j}}}=\frac{1}{\prod_{k \in \bar{j}}\left(1-z x_{k}\right)}=\sum_{s=0}^{\infty} z^{s} \chi_{s}\left(g_{\bar{j}}\right)
$$

and define the T-operators of the first level of nesting labeled by a Young diagram $\lambda=(s)$ :

$$
\mathbf{T}_{\bar{j}}^{s}(u)=\lim _{t \rightarrow \frac{1}{x_{j}}}\left(1-x_{j} t\right)(1-g t)^{\otimes N} \cdot\left[\bigotimes_{i}\left(u_{i}+2+2 \hat{D}\right) \chi_{s}\left(g_{\bar{j}}\right) w(t)\right] .
$$

The last formula also allows for an alternative to definition (12) of the Qoperators as of the T-operators for the singlet irrep in the auxiliary space corresponding to an empty Young diagram $s=0: \mathbf{Q}_{\bar{j}}(u) \equiv \mathbf{T}_{\bar{j}}^{s=0}(u)$ confirming the (more general) relation announced in (4).

In the definition (17), we take a residue of a given pole and at the same time use a character $\chi_{s}\left(g_{\bar{j}}\right)$ where one eigenvalue is "removed". This "removal" will be at the heart of our nesting procedure, and its repetition defines a certain Bäcklund flow (nesting path).

\footnotetext{
11 Which is clear in the diagonal basis since $(1-g t)^{\otimes N}$ obviously commutes with permutations, and with tensorial product of diagonal matrices, and hence with any operator of the form $\otimes_{i}\left(a_{i}+\hat{D}\right) w(b)$, due to its diagrammatic expansion given in appendix A.1
} 
Let us take the coefficient of $z^{s}\left(s \in \mathbb{Z}_{\geq 0}\right)$ in (15), analogously to what was done in (9), and rewrit 12 it, using (17), as follows:

$$
\mathbf{T}_{\bar{j}}^{s}(u) \mathbf{Q}_{\bar{\emptyset}}(u)=\mathbf{T}_{\bar{\emptyset}}^{s}(u) \mathbf{Q}_{\bar{j}}(u)-x_{j} \mathbf{T}_{\bar{\emptyset}}^{s-1}(u+2) \mathbf{Q}_{\bar{j}}(u-2),
$$

which is the simplest Baxter's TQ-relation in the operatorial form, the first of the chain of Bäcklund transformations among the commuting T- and Q-operators of the zero ${ }^{\text {th }}$ and first level of nesting known for a long time 21] on the level of their eigenvalues. Here $\mathbf{T}_{\bar{\emptyset}}^{s}(u) \equiv \mathbf{T}^{\{(s)\}}(u)$ is the T-operator of the zero ${ }^{\text {th }}$ level of nesting, the original transfer matrix (1), or (6), in the symmetric tensor irrep $\lambda=(s)$. The T- and Q-operators labeled by $\bar{j}=\mathfrak{I} \backslash\{j\}$ have $K-1$ indices, and are considered in the first level of nesting 13 .

Let us also note that the T-operators can be also defined as the residues at the poles:

$$
\begin{aligned}
\sum_{j=1}^{K} \frac{t \mathbf{T}_{\bar{j}}^{s}(u)}{1-x_{j} t}= & (1-g t)^{\otimes N} \cdot\left[\bigotimes_{i}\left(u_{i}+2+2 \hat{D}\right)\left(t \chi_{\{s\}}(g)-\chi_{\{s-1\}}(g)\right) w(t)\right] \\
& + \text { polynomial in } t .
\end{aligned}
$$

It is clear from these definitions and from (71) that all these T- (and hence the Q)operators, belong to the same family of commuting operators $\left[\mathbf{T}_{\bar{j}}^{s}(u), \mathbf{T}_{\bar{j}^{\prime}}^{s^{\prime}}\left(u^{\prime}\right)\right]=$ $\left[\mathbf{T}_{\bar{j}}^{s}(u), \mathbf{T}_{\emptyset}^{s^{\prime}}\left(u^{\prime}\right)\right]=0$. It will be also shown for all T- and Q-operators, on all levels of nesting.

4.2. Next levels of nesting . Now we will generalize this procedure, and the corresponding TQ-relations, to all nesting levels. Suppose we want to consecutively "remove" the eigenvalues $x_{j_{1}}, x_{j_{2}}, \ldots, x_{j_{k}}$ from the characters in the definition of T-operators, where $\bar{I}=\left\{j_{1}, j_{2}, \ldots, j_{k}\right\}$ is a subset of the full set of indices: $\bar{I} \subset \mathfrak{I}$ (their order is not important but they are all different). At such arbitrary level of nesting, we define a normalization operator

$$
\mathbf{B}_{\bar{I}}=\prod_{j \in \bar{I}}\left(1-x_{j} t_{j}\right) \cdot\left(1-g t_{j}\right)^{\otimes N}
$$

and the following product of generating functions of characters

$$
\Pi_{\bar{I}}=\prod_{j \in \bar{I}} w\left(t_{j}\right)
$$

The definition of the Q-operator labeled by a subset $14=\mathfrak{I} \backslash \bar{I}$ of the full set $\mathfrak{I}$ becomes

$$
\mathbf{Q}_{I}(u)=\lim _{\substack{t_{j} \rightarrow \frac{1}{x_{j}} \\ j \in \bar{I}}} \mathbf{B}_{\bar{I}} \cdot\left[\bigotimes_{i}\left(2|\bar{I}|+u_{i}+2 \hat{D}\right) \Pi_{\bar{I}}\right] \quad \text { where }|\overline{\mathrm{I}}|=\operatorname{Card}(\overline{\mathrm{I}})=\mathrm{K}-|\mathrm{I}|
$$

\footnotetext{
12 As explained above, we also use the fact that $w(z)\left(1-x_{j} z\right)=w_{\bar{j}}(z)$.

13 In the same spirit, the $k^{\text {th }}$ level of nesting will involve the quantities with $K-k$ indices.

14 the subset $I$ defines the node on the Hasse diagram where the nesting process has arrived.
} 
and once again, it is an operator on the quantum space $\left(\mathbb{C}^{K}\right)^{\otimes N}$, which is polynomial (of degree $N$ if $I \neq \emptyset$ ) in the spectral parameter $u$. Its eigenvalues have degree $\leq N$, and it can be shown (see appendix $\mathrm{B}$ ) that $\mathbf{Q}_{I}(u)\left|e_{k_{1}, k_{2}, \cdots k_{N}}\right\rangle$ is independent of all $u_{n}$ such that $k_{n} \in \bar{I}$.

We will show how to write a $T Q$ relation between the T- and Q-operators labeled by $I$ and the operators of the previous level of nesting, labeled by $I \cup j_{k}$.

Let us first generalize (16) to define the characters of $g_{I}=\operatorname{diag}\left(\left(x_{j}\right)_{j \in I}\right)$ :

$$
w_{I}(z) \equiv \operatorname{det} \frac{1}{1-z g_{I}} \equiv \prod_{j \in I} \frac{1}{1-z x_{j}} \equiv \sum_{s=0}^{\infty} z^{s} \chi_{s}\left(g_{I}\right)=\frac{w(z)}{w_{\bar{I}}(z)} .
$$

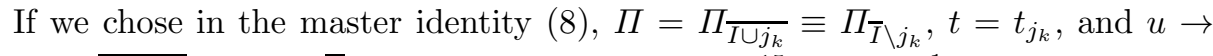
$u+2\left|\overline{I \cup j_{k}}\right|=u+2|\bar{I}|-2$, then after multiplying 15 it by $\frac{1}{w_{\bar{I} \backslash j_{k}}(z)} \mathbf{B}_{\bar{I}} \cdot \mathbf{B}_{\overline{I \cup j_{k}}}$ and taking the limit $t_{j} \rightarrow \frac{1}{x_{j}}$, we get $\lim _{\substack{t_{j} \rightarrow \frac{1}{x_{j}} \\ j \in \bar{I} \cup j_{k}}}\left(\left(1-z / t_{j_{k}}\right) \frac{\mathbf{B}_{\bar{I}}}{w_{\overline{I \cup j_{k}}}(z)} \cdot\left[\otimes_{i}\left(2|\bar{I}|+u_{i}+2 \hat{D}\right) w(z) w\left(t_{j_{k}}\right) \Pi \overline{I \cup j_{k}}\right]\right) \cdot \mathbf{Q}_{I \cup j_{k}}(u)$

$$
\begin{aligned}
& =\lim _{\substack{t_{j} \rightarrow \frac{1}{x_{j}} \\
j \in \bar{I}}}\left(\frac{\mathbf{B}_{\overline{I \cup j_{k}}}}{w_{\overline{I \cup j_{k}}}(z)} \cdot\left[\otimes_{i}\left(2|\bar{I}|-2+u_{i}+2 \hat{D}\right) w(z) \Pi \overline{\overline{I \cup j_{k}}}\right] \cdot \mathbf{Q}_{I}(u)\right. \\
& \left.-x_{j_{k}} \frac{\mathbf{B}_{\overline{I \cup j_{k}}}}{w_{\overline{I \cup j_{k}}}(z)} \cdot\left[\otimes_{i}\left(2|\bar{I}|+u_{i}+2 \hat{D}\right) z w(z) \Pi \overline{I \cup j_{k}}\right] \cdot \mathbf{Q}_{I}(u-2)\right),
\end{aligned}
$$

where $\overline{I \cup j_{k}}=\left\{j_{1}, j_{2}, \ldots, j_{k-1}\right\}$. These expressions are obtained by rewriting the $\mathrm{z}$-independent factors using the formula (22). For instance, the last factor of the last term obtained from (8) is

$$
\mathbf{B}_{\bar{I}} \cdot\left[\otimes_{i}\left(2|\bar{I}|-2+u_{i}+2 \hat{D}\right) w\left(t_{j_{k}}\right) \Pi_{\bar{I} \backslash j_{k}}\right]=\mathbf{B}_{\bar{I}} \cdot\left[\otimes_{i}\left(2|\bar{I}|-2+u_{i}+2 \hat{D}\right) \Pi_{\bar{I}}\right],
$$

which becomes $\mathbf{Q}_{I}(u-2)$ when the limit $t_{j} \rightarrow \frac{1}{x_{j}}$ is taken.

We define the T-operators for symmetric tensor representations as follows

$$
\mathbf{T}_{I}^{s}(u)=\lim _{\substack{t_{j} \rightarrow \frac{1}{x_{j}} \\ j \in \bar{I}}} \mathbf{B}_{\bar{I}} \cdot\left[\bigotimes_{i=1}^{N}\left(u_{i}+2 \hat{D}+2|\bar{I}|\right) \chi_{s}\left(g_{I}\right) \Pi_{\bar{I}}\right]
$$

where $\chi_{s}\left(g_{I}\right)$ is defined by (23). Then the action of co-derivatives on $\chi_{s}\left(g_{I}\right)$ is a priori rather nontrivial. The recipe to avoid this complication and compute

\footnotetext{
15 Once again, the normalization factors $\mathbf{B}_{\bar{I}}$ and $\mathbf{B}_{\overline{I \cup j_{k}}}$ commute with all the other factors, because they commute with all permutations, and with all operators $g^{i_{1}} \otimes g^{i_{2}} \otimes \cdots g^{i_{N}}$, which are the building blocks of all other factors.

On the other hand, we will see a posteriori in appendix $\mathrm{F}$ that the factor $w_{\bar{I}}(z)$ can be freely move across the $\hat{D}$ 's.
} 
T-operators is given in appendix $\mathbf{F}$ In terms of the generating function of $\mathbf{T}_{I}^{s}(u)$ we have

$$
\begin{aligned}
\mathfrak{W}_{I}(u, z) \equiv \sum_{s=0}^{\infty} z^{s} \mathbf{T}_{I}^{s}(u) & =\lim _{\substack{t_{j} \rightarrow \frac{1}{x_{j}} \\
j \in \bar{I}}} \mathbf{B}_{\bar{I}} \cdot\left[\bigotimes_{i=1}^{N}\left(u_{i}+2 \hat{D}+2|\bar{I}|\right) \frac{w(z)}{w_{\bar{I}}(z)} \Pi_{\bar{I}}\right] \\
& =\frac{1}{w_{\bar{I}}(z)} \lim _{\substack{t_{j} \rightarrow \frac{1}{x_{j}} \\
j \in \bar{I}}} \mathbf{B}_{\bar{I}} \cdot\left[\bigotimes_{i=1}^{N}\left(u_{i}+2 \hat{D}+2|\bar{I}|\right) w(z) \Pi_{\bar{I}}\right] .
\end{aligned}
$$

Finally, we can notice that the L.H.S. of (24) contains $\frac{\left(1-z x_{j_{k}}\right)}{w_{\overline{I \cup j_{k}}}(z)}=\frac{1}{w_{\bar{I}}(z)}$. Then, expanding (24) with respect to $z$ and taking the coefficients of $z^{s}$, we obtain using the definition (28) the following operatorial TQ-relation

$$
\mathbf{T}_{I}^{s}(u) \mathbf{Q}_{I \cup j_{k}}(u)=\mathbf{T}_{I \cup j_{k}}^{s}(u) \mathbf{Q}_{I}(u)-x_{j_{k}} \mathbf{T}_{I \cup j_{k}}^{s-1}(u+2) \mathbf{Q}_{I}(u-2) .
$$

It generalizes the similar, obvious relation among the characters of symmetric tensor irreps:

$$
\chi_{s}\left(g_{I}\right)=\chi_{s}\left(g_{I, j}\right)-x_{j} \chi_{s-1}\left(g_{I, j}\right),
$$

where $j \in \bar{I}$ and $s \in \mathbb{Z}_{\geq 1}$.

Notice that again the Q-operator on any level of nesting is equal to the Toperator, with the same index set $I$, for an empty Young diagram $\lambda=\emptyset$ (which corresponds to $s=0$ case):

$$
\mathbf{Q}_{I}(u)=\mathbf{T}_{I}^{\{\emptyset\}}(u)
$$

4.3. Generalization to any representations. There is a natural way to generalize the T-operators to any irreps $\lambda$ in the auxiliary space:

$$
\mathbf{T}_{I}^{\{\lambda\}}(u)=\lim _{\substack{t_{j} \rightarrow \frac{1}{x_{j}} \\ j \in \bar{I}}} \mathbf{B}_{\bar{I}} \cdot\left[\bigotimes_{i=1}^{N}\left(u_{i}+2 \hat{D}+2|\bar{I}|\right) \chi_{\lambda}\left(g_{I}\right) \Pi_{\bar{I}}\right]
$$

where the $g l(K-|\bar{I}|)$ characters of the irreps $\lambda$ are given through the characters of the symmetric tensor representations $\chi_{s}\left(g_{I}\right)$ by the Jacobi-Trudi determinant formula

$$
\chi_{\{\lambda\}}\left(g_{I}\right)=\operatorname{det}_{1 \leq i, j \leq a} \chi_{\lambda_{j}+i-j}\left(g_{I}\right),
$$

where $a$ is the number of rows in the Young diagram $\lambda$. It is noteworthy that, due to the definition (32), $\mathbf{T}_{I}^{\{\lambda\}}=0$ if $\lambda$ has more than $|I|$ rows, because $\chi_{\{\lambda\}}\left(g_{I}\right)=0$.

The Bazhanov-Reshetikhin (BR) formula proven in [1 at the zero ${ }^{\text {th }}$ level of nesting is also true for the T-operators on every level of nesting, and it reads

$$
\mathbf{T}_{I}^{\left\{\left(\lambda_{1}, \lambda_{2}, \cdots, \lambda_{a}\right)\right\}}(u)=\frac{1}{\prod_{k=1}^{a-1} \mathbf{Q}_{I}(u-2 k)} \operatorname{det}_{1 \leq i, j \leq a}\left(\mathbf{T}_{I}^{\lambda_{j}+i-j}(u+2-2 i)\right) .
$$


In the particular case of the rectangular Young diagrams $\lambda=\left(s^{a}\right)$ this means that the T-operators satisfy the immediate equivalent of the BR formula - the Hirota equation 16 , which is the same on any level of nesting, namely

$$
\begin{aligned}
\mathbf{T}_{I}^{(a, s)}(u+1) \mathbf{T}_{I}^{(a, s)}(u-1)=\mathbf{T}_{I}^{(a+1, s)} & (u+1) \mathbf{T}_{I}^{(a-1, s)}(u-1) \\
& +\mathbf{T}_{I}^{(a, s+1)}(u-1) \mathbf{T}_{I}^{(a, s-1)}(u+1) .
\end{aligned}
$$

It can be proven that it is a consequence of the equation 866 of appendix E. It is also well known that the TQ-relation (29) implies the generating series expression (75) of T-operators for symmetric representations in terms of Q-operators, detailed in the appendix C] (see for instance 21 at the level of eigenvalues). Then the Bazhanov-Reshetikhin determinant formula (or, equivalently, the Hirota equation) allows to write arbitrary $\mathbf{T}_{I}^{\{\lambda\}}(u)$ operators in terms of Q-operators and to check the following bilinear relations on the nested Toperators 21] (see 22] for the case with non-zero twist $g$ ):

$$
\begin{aligned}
\mathbf{T}_{I, j}^{(a+1, s)}(u) \mathbf{T}_{I}^{(a, s)}(u)-\mathbf{T}_{I, j}^{(a, s)}(u) & \mathbf{T}_{I}^{(a+1, s)}(u) \\
& =x_{j} \mathbf{T}_{I, j}^{(a+1, s-1)}(u+2) \mathbf{T}_{I}^{(a, s+1)}(u-2), \\
\mathbf{T}_{I, j}^{(a, s+1)}(u) \mathbf{T}_{I}^{(a, s)}(u)-\mathbf{T}_{I, j}^{(a, s)}(u) & \mathbf{T}_{I}^{(a, s+1)}(u) \\
= & x_{j} \mathbf{T}_{I, j}^{(a+1, s)}(u+2) \mathbf{T}_{I}^{(a-1, s+1)}(u-2) .
\end{aligned}
$$

The TQ-relation (29) is a particular case of equation (37) when $a=0$, and the two equations (37, 38) coincide with the definition of Bäcklund flow given in 22 . up to the permutations on the index set $I$, so that the definitions (31) 32) for nested T- and Q-operators explicitly give a solution of the linear system (37. 38).

4.4. QQ-relations. In the previous subsections, the TQ-relations were proven from the formula (8). This formula contains the explicit factors $w(z)$ and $w(t)$, and one of them was incorporated into $\Pi$ (by defining $\Pi_{\overline{I \cup j_{k}}}=w(t) \Pi_{\bar{I}}$ ) and was associated to the nesting level. The other factor $\left(w(z)=\sum z^{s} \chi_{s}\right)$ was decomposed into the characters of $s$-symmetric representations.

16 Hirota relation can also be written in terms of the T-operators defined as $\mathbb{T}_{a, s}(u)=$ $\mathbf{T}^{(a, s)}(u+a-s)$. In term of these $\mathbb{T}$-operators, 35 takes the usual form $\mathbb{T}_{a, s}(u+1) \mathbb{T}_{a, s}(u-1)=$ $\mathbb{T}_{a+1, s}(u) \mathbb{T}_{a-1, s}(u)+\mathbb{T}_{a, s+1}(u) \mathbb{T}_{a, s-1}(u)$, more frequent in the literature.

17 To prove the BR at arbitrary levels of nesting, one actually needs to rewrite [86] in the following slightly more general form, which is also equivalent to (8):

$$
\mathbf{W}_{I, J}(u)=\frac{\operatorname{det}\left(z_{j}^{n-k} \mathbf{W}_{I, j}(u-2 k+2)\right)_{\substack{j \in J \\ 1 \leq k \leq|J|}}}{\operatorname{det}\left(z_{j}^{n-k}\right)_{\substack{j \in J \\ 1 \leq k \leq|J|}} \prod_{k=1}^{|J|-1} \mathbf{W}_{I}(u-2 k)},
$$

which becomes the nested Bazhanov-Reshetikhin formula after the limit $z_{i} \rightarrow 1 / x_{i}$ is taken for $i \in I$. 
But it is also possible to generate other identities from (8), for instance by incorporating both $w(t)$ and $w(z)$ into $\Pi_{\bar{I}}$, which gives rise to different nesting paths. Then, a careful rewriting 18 of (8) immediately gives the following well known QQ-relations between Q-operators:

$$
\left(x_{i}-x_{j}\right) \mathbf{Q}_{I}(u-2) \mathbf{Q}_{I, i, j}(u)=x_{i} \mathbf{Q}_{I, j}(u-2) \mathbf{Q}_{I, i}(u)-x_{j} \mathbf{Q}_{I, j}(u) \mathbf{Q}_{I, i}(u-2),
$$

where $i, j \in \bar{I}$ and $i \neq j$. These are relations among Q-operators in 4-cycles made of $\left\{\mathbf{Q}_{I}, \mathbf{Q}_{I, i}, \mathbf{Q}_{I, j}, \mathbf{Q}_{I, i, j}\right\}$ in the Hasse diagram (cf. figure 1 ).

We can immediately solve these QQ-relations, which are actually a particular case of the Plücker identities for all the Q-operators to get the following determinant representations 19

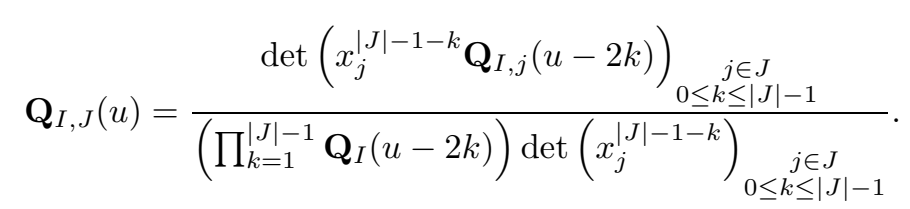

In particular, choosing $I=\emptyset$ gives the expression of any Q-operator in terms of $K+1$ Q-operators, namely, the $K$ single indexed $\mathbf{Q}_{i}(u)$ operators describing the last level of nesting, and the $u$-independent operator $\mathbf{Q}_{\emptyset}(u)$. More explicitly $\mathbf{Q}_{\emptyset}(u)$ can be defined by its action on the diagonal basis of the quantum space by

$$
\mathbf{Q}_{\emptyset}(u)|e\rangle \equiv \mathbf{T}_{\emptyset}^{s=0}(u)|e\rangle=2^{N} \prod_{k=1}^{K} n_{k} ! \prod_{\substack{j=1,(j \neq k)}}^{K}\left(1-\frac{x_{j}}{x_{k}}\right)^{n_{j}-1}|e\rangle,
$$

where $|e\rangle=\left|e_{i_{1}, i_{2}, \cdots, i_{N}}\right\rangle \equiv\left|e_{i_{1}}\right\rangle \otimes\left|e_{i_{2}}\right\rangle \otimes \cdots \otimes\left|e_{i_{N-1}}\right\rangle \otimes\left|e_{i_{N}}\right\rangle$ and $n_{k}$ is the number of $j$ such that $i_{j}=k$.

4.5. Operatorial Bethe equations. In this subsection we derive the set of nested Bethe ansatz equations.

From the QQ-relations (39) one immediately sees that since $\mathbf{Q}_{I, j}(u)$ should be, by its definition, a polynomial of $u$, then (for $i, j \in \bar{I}$ and $i \neq j$ )

$$
\begin{aligned}
& \mathbf{Q}_{I, i}(u) \mid\left(x_{i}-x_{j}\right) \mathbf{Q}_{I}(u-2) \mathbf{Q}_{I, i, j}(u)+x_{j} \mathbf{Q}_{I, j}(u) \mathbf{Q}_{I, i}(u-2), \\
& \mathbf{Q}_{I, i}(u) \mid\left(x_{i}-x_{j}\right) \mathbf{Q}_{I}(u) \mathbf{Q}_{I, i, j}(u+2)-x_{i} \mathbf{Q}_{I, j}(u) \mathbf{Q}_{I, i}(u+2),
\end{aligned}
$$

where $P \mid P^{\prime}$ denotes the fact that the polynomial $P^{\prime}$ contains the polynomial $P$ as a factor. By adding $x_{i} \mathbf{Q}_{I, i}(u+2)$ times the first line to $x_{j} \mathbf{Q}_{I, i}(u-2)$ times the second line, one gets

$$
\mathbf{Q}_{I, i}(u) \mid x_{i} \mathbf{Q}_{I}(u-2) \mathbf{Q}_{I, i, j}(u) \mathbf{Q}_{I, i}(u+2)+x_{j} \mathbf{Q}_{I}(u) \mathbf{Q}_{I, i, j}(u+2) \mathbf{Q}_{I, i}(u-2) .
$$

\footnotetext{
18 In (8), let us put $t=t_{j}, z=t_{i}, \Pi=\Pi \overline{I \cup\{i, j\}}$, where $I \subset \mathfrak{I}$ and $i, j \in \bar{I}(i \neq j)$. Then note the following relations: $w(z) w(t) \Pi=\Pi_{\bar{I}}, w(z) \Pi=\Pi \overline{I \cup\{j\}}, w(t) \Pi=\Pi \overline{I \cup\{i\}}$.

19 The factor $\operatorname{det}\left(x_{j}^{|J|-1-k}\right)_{\substack{j \in J \leq J \mid-1 \\ 0 \leq k \leq J}}=\prod_{i<j ; i, j \in J}\left(x_{i}-x_{j}\right)$ in the denominator corresponds to the denominator formula of the character of $g l(|J|)$.
} 
This is written for the Q-operators, but when acting on particular eigenstates, the operatorial Bethe equations (44) become the usual polynomial Bethe equations (46) [28, 29] (cf. eq. (68) of [22]) on the Bethe roots $\left\{u_{k}^{(I)}\right\}$ along a chosen nesting path. Indeed, now we know, by construction, that the Q-operators are polynomial 20 of $u$ and therefore for each eigenstate their eigenvalues are also polynomials of a degree $K_{I} \leq N$ in $u$ :

$$
\mathrm{Q}_{I}(u)=c_{I} \prod_{k=1}^{K_{I}}\left(u-u_{k}^{(I)}\right)
$$

so that, substituting $u=u_{k}^{(I, i)}$ into an eigenvalue of (44), we obtain the usual nested Bethe ansatz equations:

$$
-1=\frac{x_{i}}{x_{j}} \frac{\mathrm{Q}_{I}\left(u_{k}^{(I, i)}-2\right) \mathrm{Q}_{I, i}\left(u_{k}^{(I, i)}+2\right) \mathrm{Q}_{I, i, j}\left(u_{k}^{(I, i)}\right)}{\mathrm{Q}_{I}\left(u_{k}^{(I, i)}\right) \mathrm{Q}_{I, i}\left(u_{k}^{(I, i)}-2\right) \mathrm{Q}_{I, i, j}\left(u_{k}^{(I, i)}+2\right)} \quad \text { for } \quad k \in\left\{1,2, \ldots, K_{I, i}\right\} .
$$

The Q-operators are formal polynomials $\mathbf{Q}_{I}(u)=\sum_{k=1}^{N} u^{k} \mathbf{c}_{k}^{(I)}$ whose coefficients $\left\{\mathbf{c}_{k}^{(I)}\right\}$ are also operators in the quantum space. On particular quantum states - the eigenvectors of the spin chain Hamiltonian, a part of them becomes zero, which explains the fact that the power of Q-functions - the eigenvalues of the Q-operators - can diminish on each step of the Bäcklund procedure.

Given all solutions of the Bethe equations (46) it is possible, in principle, to find all eigenvalues of the $\mathbf{Q}_{I_{j}}$ operators and then to reconstruct all T-operators using TQ-relations (see (75) ) together with the Hirota relation.

\section{Generalization to the supersymmetric case}

In the case of the $g l(K \mid M)$ super-spin chain, T- and Q-operators are labelled by the $2^{K+M}$ subsets $I$ of the full set $\mathfrak{I}=\{1,2, \ldots, K+M\}$. For any element of $\mathfrak{I}$, we define the grading parameter:

$$
p_{b}=0 \quad \text { for } \quad 1 \leq b \leq K, \quad \text { and } \quad p_{f}=1 \quad \text { for } \quad K+1 \leq f \leq K+M .
$$

Now the Q-operators are described by the colored Hasse diagram (see figure 2). by

In the case of the $g l(K \mid M)$ super-spin chain, the co-derivative can be defined

$$
\hat{D} \otimes f(g)=\sum_{i j} e_{i j} \frac{\partial}{\partial \phi_{i}^{j}} \otimes f\left(e^{\sum_{k l} \phi_{l}^{k} e_{k l}} g\right)_{\phi=0}, \quad \frac{\partial}{\partial \phi_{i_{1}}^{j_{1}}} \phi_{j_{2}}^{i_{2}} \equiv \delta_{j_{1}}^{i_{2}} \delta_{j_{2}}^{i_{1}}(-1)^{p_{j_{1}}}
$$

where $\left\{e_{i j}\right\}$ are generators of $g l(K \mid M)$ in the fundamental representation and a matrix $\phi$ is expressed as $\phi=\sum \phi_{j}^{i} e_{i j}$, and $g$ is a matrix in the fundamental representation of $G L(K \mid M)$.

20 This fact was missing in the analytic Bethe ansatz construction of 22 and appeared there only as a hypothetic ansatz for the solution of Hirota equation by the Bäcklund procedure. 


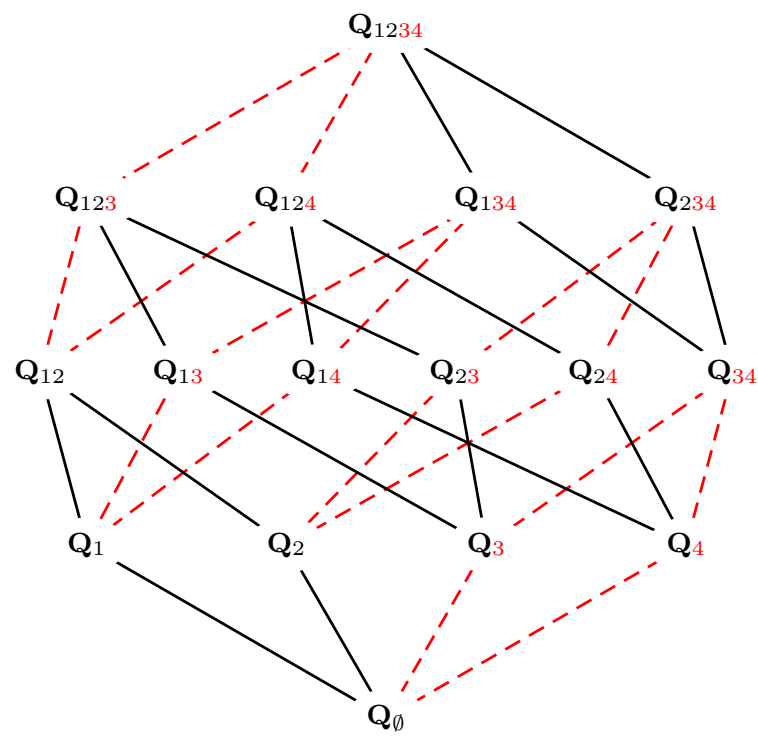

Fig. 2: Hasse diagram for the Q-operators: $g l(2 \mid 2)$ case. There are $2^{4}=16$ Qoperators in the same way as $g l(4)$ case (figure $1 \mathrm{~b}) . \mathbf{Q}_{I}$ and $\mathbf{Q}_{I \backslash\{k\}}$ are connected by a solid line if $k \in\{1,2\}$ (bosonic $p_{k}=0$ ), dashed line if $k \in\{3,4\}$ (fermionic $\left.p_{k}=1\right)$.

As explained in [1, the properties of co-derivatives are exactly the same as in the bosonic case, including the expression of T-operators in terms of coderivatives (at the zero ${ }^{\text {th }}$ nesting level). The diagrammatics of the co-derivative is also the same as in the bosonic case, except the signs to be introduced into every permutation operator, to get $\mathcal{P}=\sum_{\alpha \beta}(-1)^{p_{\alpha}} e_{\beta \alpha} \otimes e_{\alpha \beta}$. In particular, the formula (8) still holds in the super-case but (7) has to be substituted by

$w(z)=\operatorname{sdet} \frac{1}{1-z g}=\frac{\prod_{j=1}^{M}\left(1-z y_{j}\right)}{\prod_{j=1}^{K}\left(1-z x_{j}\right)}=\sum_{s=0}^{\infty} z^{s} \chi_{s}(g)=\frac{1}{\sum_{a=0}^{\infty}(-1)^{a} z^{a} \chi^{(a)}(g)}$,

where $\left(x_{1}, \cdots, x_{K}, y_{1}, \cdots, y_{M}\right) \equiv\left(\xi_{1}, \cdots, \xi_{K+M}\right)$ are the eigenvalues of $g \in$ $G L(K \mid M)$ in the fundamental representation and sdet denotes the super-determinant. With slight generalizations of the definitions w.r.t. the bosonic case, all the supersymmetric TQ- and QQ-relations follow from (8) if we define T- and Q-operators in the following way:

$$
\Pi_{\bar{I}}=\prod_{j \in \bar{I}} w\left(t_{j}\right)^{\left((-1)^{p_{j}}\right)}, \quad \mathbf{B}_{\bar{I}}=\prod_{j \in \bar{I}}\left(1-\xi_{j} t_{j}\right)\left(1-g t_{j}\right)^{\otimes N},
$$




$$
\begin{aligned}
& \mathbf{T}_{I}^{\{\lambda\}}(u)=\lim _{\substack{t_{j} \rightarrow \frac{1}{\xi_{j}} \\
j \in \bar{I}}} \mathbf{B}_{\bar{I}} \cdot\left[\bigotimes_{i=1}^{N}\left(u_{i}+2 \hat{D}+2 n_{\bar{b}}-2 n_{\bar{f}}\right) \chi_{\lambda}\left(g_{I}\right) \Pi_{\bar{I}}\right], \\
& \mathbf{Q}_{I}(u)=\lim _{\substack{t_{j} \rightarrow \frac{1}{\xi_{j}} \\
j \in \bar{I}}} \mathbf{B}_{\bar{I}} \cdot\left[\bigotimes_{i=1}^{N}\left(u_{i}+2 \hat{D}+2 n_{\bar{b}}-2 n_{\bar{f}}\right) \Pi_{\bar{I}}\right]
\end{aligned}
$$

where $n_{\bar{b}}=|\bar{I} \cap\{1,2, \cdots, K\}|$, and ${ }^{21} n_{\bar{f}}=|\bar{I} \cap\{K+1, \cdots, K+M\}|$. In particular, the Q-operator for an empty set (41) now becomes

$$
\mathbf{Q}_{\emptyset}(u)|e\rangle \equiv \mathbf{T}_{\emptyset}^{\{\emptyset\}}(u)|e\rangle=2^{N} \prod_{k=1}^{K+M}(-1)^{p_{k} n_{k}} n_{k} ! \prod_{\substack{j=1,(j \neq k)}}^{K+M}\left(1-\frac{\xi_{j}}{\xi_{k}}\right)^{n_{j}-(-1)^{p_{j}+p_{k}}}|e\rangle
$$

In what follows, the indices $i, j \in\{1,2, \ldots, K\} \cap \bar{I}$ and $\hat{k}, \hat{l} \in\{K+1, K+$ $2, \ldots, K+M\} \cap \bar{I}$ will correspond to the opposite gradings (we might call $i$ and $j$ "bosons" and $k, l$ "fermions"), and we will use the notation $\hat{l}=l+K$ for $l \in\{1,2, \ldots, M\}$, so that $\xi_{\hat{l}}=y_{l}$. In (51), $\chi_{\lambda}\left(g_{I}\right)$ is defined through (33), where $\chi_{s}\left(g_{I}\right)$ is defined by the generating series

$$
w_{I}(z) \equiv \operatorname{sdet} \frac{1}{1-z g_{I}}=\frac{\prod_{\substack{\hat{l} \in I \\ l>K}}\left(1-z y_{l}\right)}{\prod_{\substack{j \in I \\ j \leq K}}\left(1-z x_{j}\right)}=\sum_{s=0}^{\infty} z^{s} \chi_{s}\left(g_{I}\right)
$$

That implies [30,7] that $\mathbf{T}_{\mathfrak{I}}^{\{\lambda\}}=0$ if the Young diagram $\lambda=\left(\lambda_{1}, \lambda_{2}, \lambda_{3} \ldots\right)$ contains the highest weight $\lambda_{K+1}>M$ (i.e. unless $\lambda$ is inside the "fat hook region" indicated in figure 10 in [1]), while for nested T-operators $\mathbf{T}_{I}^{\{\lambda\}}$, the same "fat-hook condition" holds, but the corner of the fat hook is displaced from $(M, K)$ to $\left(n_{f}, n_{b}\right)$, where $n_{b}=|I \cap\{1,2, \cdots, K\}|, \quad n_{f}=\mid I \cap\{K+$ $1, \cdots, K+M\} \mid$. This means that the "hook region" decreases by one row or one column at each level of nesting.

Then the TQ relations (29) become

$$
\begin{gathered}
\mathbf{T}_{I}^{s}(u) \mathbf{Q}_{I, j}(u)=\mathbf{T}_{I, j}^{s}(u) \mathbf{Q}_{I}(u)-x_{j} \mathbf{T}_{I, j}^{s-1}(u+2) \mathbf{Q}_{I}(u-2) \\
\mathbf{T}_{I, \hat{l}}^{s}(u) \mathbf{Q}_{I}(u)=\mathbf{T}_{I}^{s}(u) \mathbf{Q}_{I, \hat{l}}(u)-y_{l} \mathbf{T}_{I}^{s-1}(u+2) \mathbf{Q}_{I, \hat{l}}(u-2) \\
\text { for } 1 \leq j \leq K \text { and } K+1 \leq \hat{l} \leq K+M
\end{gathered}
$$

\footnotetext{
21 As before, $|I|$ denotes $\operatorname{Card}(I)$

22 obtained from the master identity (8) by putting $\Pi=\Pi \overline{I \cup\{j\}}, t=t_{j}$ for 5 (55); $\Pi=$ $\Pi_{\bar{I}}, t=t_{\hat{j}}$ for $(56)$.
} 
The QQ-relation:23 also become grading-dependent:

$$
\begin{gathered}
\left(x_{i}-x_{j}\right) \mathbf{Q}_{I}(u-2) \mathbf{Q}_{I, i, j}(u)=x_{i} \mathbf{Q}_{I, j}(u-2) \mathbf{Q}_{I, i}(u)-x_{j} \mathbf{Q}_{I, j}(u) \mathbf{Q}_{I, i}(u-2), \\
\left(x_{i}-y_{l}\right) \mathbf{Q}_{I, \hat{l}}(u-2) \mathbf{Q}_{I, i}(u)=x_{i} \mathbf{Q}_{I}(u-2) \mathbf{Q}_{I, i, \hat{l}}(u)-y_{l} \mathbf{Q}_{I}(u) \mathbf{Q}_{I, i, \hat{l}}(u-2), \\
\left(y_{l}-y_{m}\right) \mathbf{Q}_{I, \hat{l}, \hat{m}}(u-2) \mathbf{Q}_{I}(u)=y_{l} \mathbf{Q}_{I, \hat{l}}(u-2) \mathbf{Q}_{I, \hat{m}}(u)-y_{m} \mathbf{Q}_{I, \hat{l}}(u) \mathbf{Q}_{I, \hat{m}}(u-2),
\end{gathered}
$$

$$
\text { for } \quad i, j \in\{1,2, \ldots, K\} \cap \bar{I} \quad \text { and } \quad \hat{l}, \hat{m} \in\{K+1, K+2, \ldots, K+M\} \cap \bar{I} .
$$

These are relations among Q-operators in 4-cycles made of $\left\{\mathbf{Q}_{I}, \mathbf{Q}_{I, i}, \mathbf{Q}_{I, j}, \mathbf{Q}_{I, i, j}\right\}$ in the Hasse diagram fig. 2. For example, in the figure 2, eq. 577 corresponds to 4-cycles made of 4-solid lines, (58) corresponds to 4-cycles made of 2-solid lines and 2-dashed lines, (59) corresponds to 4-cycles made of 4-dashed lines.

All these TQ- and QQ- relations are derived by choosing an appropriate $\Pi$ in (8), but, as explained in 31, they could have been obtained from the bosonic relations by the so called "bosonization trick" : For instance, (58) can be rewritten as

$$
\left(x_{i}-y_{l}\right) \mathbf{Q}_{J}(u-2) \mathbf{Q}_{J, i \backslash \hat{l}}(u)=x_{i} \mathbf{Q}_{J \backslash \hat{l}}(u-2) \mathbf{Q}_{J, i}(u)-y_{l} \mathbf{Q}_{J \backslash \hat{l}}(u) \mathbf{Q}_{J}(u-2),
$$

where $J=I \cup\{\hat{l}\}$, which has exactly the same form as (57), up the the formal replacement $J \rightarrow I, J \backslash \hat{l} \rightarrow I, l$. This is interpreted as the fact that adding a "boson" to the set $I$ (which indexes Q or T-operators) is equivalent to removing a "fermion" 24. This trick can be viewed as a mnemonic rule, and in this construction of the T- and Q-operators, it comes 25 from the power $(-1)^{p_{j}}$ in the definition (50) of $\Pi_{\bar{I}}$. This trick means that the Hasse diagram for $g l(K \mid M)$ can be "rotated" by putting $\mathbf{Q}_{K+1, K+2, \cdots, K+M}$ on the top row and $\mathbf{Q}_{1,2, \cdots, K}$ on the bottom row, so that the QQ-relations take the same form for all the facets of the modified diagram.

In the same way as for the $g l(K)$ case, the TQ-relations (55, 56) can be written as the generating series expansion (78) (see 20,22 at the level of eigenvalues). We can also generalize the Wronskian expressions (40) for Q-operators to all the T-operators at all levels of nesting. They do not differ in the form from the relations found in 23 .

The Bethe ansatz equations for $g l(K \mid M)$ 28 are obtained in the same way as for the $g l(K)$ case: there are two "bosonic" Bethe Ansatz equations (BAEs)

\footnotetext{
23 obtained from the master identity (8) by putting $\Pi=\Pi \overline{I \cup\{i, j\}}, t=t_{j}, z=t_{i}$ for (57); $\Pi=\Pi \frac{\overline{I \cup\{i\}}}{\Pi}, t=t_{\hat{l}}, z=t_{i}$ for (58); $\Pi=\Pi_{\bar{I}}, t=t_{\hat{m}}, z=t_{\hat{l}}$ for (59).

24 As explained in 22], the same linear system describes the addition of a column to the "fat hook" or the removal of a line (see (41) and (42) in 20 22 ). In our notation, the former case is the addition of a "fermion" to the set I, while the latter is the removal of a "boson".

25 In (50:52) we can see that adding a "boson" to the set $I$ multiplies $\Pi_{\bar{T}}$ by $w\left(t_{j}\right)$, while adding a "fermion" divides it by $w\left(t_{j}\right)$. As the "Master Identity" is only sensible to the addition/removal of $w\left(t_{j}\right)$ factors, it is not surprising that the QQ-relation obtained from "master identity" satisfies this "bosonization trick."
} 
and two "fermionic" ones 26 . The first "bosonic" BAE follows from (57) and is unchanged with respect to the section 4.5: eq. (44) on the level of the operator and eq. (46) on the level of the eigenvalue. The second bosonic BAE is obtained by isolating $\mathbf{Q}_{I, \hat{l}}$ in (59) which gives

$$
\mathbf{Q}_{I, \hat{l}}(u) \mid y_{m} \mathbf{Q}_{I, \hat{l}, \hat{m}}(u-2) \mathbf{Q}_{I}(u) \mathbf{Q}_{I, \hat{l}}(u+2)+y_{l} \mathbf{Q}_{I, \hat{l}, \hat{m}}(u) \mathbf{Q}_{I}(u+2) \mathbf{Q}_{I, \hat{l}}(u-2),
$$

which is, at the level of eigenvalues, the equation (81), equivalent to the equation (69) of [22] (up to the permutation on the indices (the Weyl group symmetry)).

On the other hand, the "fermionic" BAEs can be immediately obtained from (58) in the form

$$
\begin{aligned}
& \mathbf{Q}_{I, i}(u) \mid x_{i} \mathbf{Q}_{I}(u-2) \mathbf{Q}_{I, i, \hat{l}}(u)-y_{l} \mathbf{Q}_{I}(u) \mathbf{Q}_{I, i, \hat{l}}(u-2), \\
& \mathbf{Q}_{I, \hat{l}}(u) \mid x_{i} \mathbf{Q}_{I}(u) \mathbf{Q}_{I, i, \hat{l}}(u+2)-y_{l} \mathbf{Q}_{I}(u+2) \mathbf{Q}_{I, i, \hat{l}}(u) .
\end{aligned}
$$

In terms of eigenvalues, the Bethe equation (62) (resp (63)) is written as (82) (resp (83)) in the appendix D.

\section{Conclusions}

The co-derivative formalism and the master identity (8), together with the definitions (22), (32) of nested T- and Q-operators proposed in this paper can serve as an alternative approach to the quantum integrability, rather different from the popular algebraic Bethe ansatz (see for example 32 and the references therein). It allows to complete the whole procedure of diagonalization of transfer-matrix of the inhomogeneous twisted $g l(K \mid M)$ (super)spin chain, all the way from its construction from R-matrices obeying the Yang-Baxter relations and till the nested system of Bethe ansatz equations, directly in terms of the operators acting on the quantum space. The master identity (8) presented at the beginning of the paper and generalizing a similar identity from [1] is the basis of this approach, encoding all possible operatorial QQ- and Baxter's TQ-relations at every step of nesting, or of the operatorial Bäcklund flow, generalizing the operatorial Bäcklund transformations of [21,20]. Remarkably, the master identity takes a bilinear form with respect to the $g l(K \mid M)$ characters, or their generating functions. Since the characters can be viewed as the tau-functions of KdV hierarchy (which is of course a reduction of KP hierarchy) one can speculate that this identity is simply a particular case of the general Hirota identity for the KP tau-functions, with $\tau_{n}=\frac{1}{n} \operatorname{tr} g^{n}$ playing the role of the KP "times". It would be an interesting relation between the quantum and classical integrability, showing that, paradoxically, the former is a particular case of the latter.

It would be also interesting to generalize our approach to the case of noncompact representations of $g l(K \mid M)$ in the auxiliary space, following the observations made in $33,31,34$ for the characters and Q-operators for $U(2,2 \mid 4)$. This might teach us how to deal with one of the most interesting integrable

\footnotetext{
26 We will call "bosonic" (resp "fermionic") the Bethe Ansatz Equations having two free indices of same grading (resp of opposite grading). For instance, in (61), the two free indices $\hat{l}$ and $\hat{m}$ have the same grading, hence this Bethe equation is called "bosonic".
} 
physical systems, $\mathcal{N}=4$ SYM theory and its AdS dual - the Metsaev-Tseytlin sigma-model having the $P S U(2,2 \mid 4)$ global symmetry. In general, the Y-systems for sigma-models and their Wronskian solutions [8,9] might be also an interesting subject for their operatorial generalization in the quantum (physical) space and might give us an interesting tool for the study of the spectrum of excited states and shed some light on the formulas for the energy of an excited state conjectured in the literature for relativistic sigma models [35, 36, 9] and for the AdS/CFT [37. It would be also interesting to generalize our master identity to other symmetries of the spin chains, where the Bazhanov-Reshetikhin-type relations are also known, to other than fundamental irreps in the quantum space, as well as to the trigonometric and elliptic R-matrices.

\section{Acknowledgments}

The work of VK was partly supported by the grant RFFI 08-02-00287. The work of VK was also partly supported by the ANR grant GranMA (BLAN-081-313695). We thank A. Alexandrov, V. Bazhanov, L. Février, N. Gromov, T. Lukowski, C. Meneghelli, M. Staudacher, P. Vieira and especially A. Zabrodin for useful comments and discussions. The work of ZT was supported by Nishina Memorial Foundation and by Grant-in-Aid for Young Scientists, B \#19740244 from The Ministry of Education, Culture, Sports, Science and Technology in Japan. ZT thanks École Normale Superieure, LPT, where a considerable part of this work was done, for the kind hospitality.

\section{Note added}

When finishing this paper we learned about the results of 19 which deal with the same problem, the operatorial formulation of the Q-operators and of the TQ-relations. The objects studied in that forthcoming paper are the same, but the formalism is radically different from ours.

\section{Notes for version 2}

We corrected in this version [arXiv:1010.4022v2 [math-ph]] many minor misprints of the first version, and added some details and explanations. It is precised that $\Pi(g)$ in the master identity (8) has a form of det $f(g)$, where $f(z)$ is an arbitrary fixed function, rather than an arbitrary class function of the twist matrix $g$. We also removed a false TT-relation (eq. (4.27) in the version 1).

Most importantly, we give in this new version a concise proof of our master identity - the basis of our approach.

\section{Notes for version 3}

Minor misprints are corrected. 


\section{A. Diagrammatics of co-derivatives}

A.1. Co-Derivatives and characters. The action of the co-derivative on characters and their generating function is explained in [1. For instance, we can write

$\hat{D} \otimes \hat{D} \quad w(x)=\frac{g x}{1-g x} \otimes \frac{g x}{1-g x}+\mathcal{P}_{1,2}\left(\frac{1}{1-g x} \otimes \frac{g x}{1-g x}\right)=(\lfloor+\grave{\jmath}) w(x)$,

where $\left\lfloor\left(\right.\right.$ resp $\vdots$ ) stands for $\frac{g x}{1-g x}\left(\operatorname{resp} \frac{1}{1-g x}\right)$, so that $\left\lfloor=\frac{g x}{1-g x} \otimes \frac{g x}{1-g x}\right.$ and $\therefore=\mathcal{P}_{1,2}\left(\frac{1}{1-g x} \otimes \frac{g x}{1-g x}\right)$, where $\mathcal{P}_{1,2}$ denotes the usual permutation between the $1^{\text {st }}$ and $2^{\text {nd }}$ quantum space 27 . The general expression for $\hat{D}^{\otimes N} w(x)$ is given by the formula (4.11) of [1] (formula (30) in the arXiv version), and it represents the sum of diagrams corresponding to all possible permutations, with dashing all the lines going from lower to upper nodes and directed to the right.

In terms of characters of the symmetric tensor irreps $\chi_{s}(g)$, the equation (64) reads

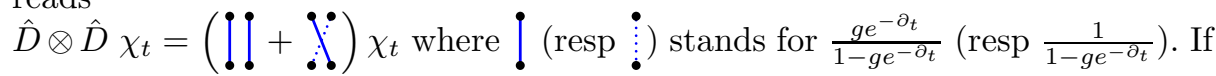
we identify $x=e^{-\partial_{t}}$ there is no ambiguity between these two definitions of the diagrammatics.

This diagrammatics can also be extended for the inclusion of the parameters $u_{i}$, i.e.

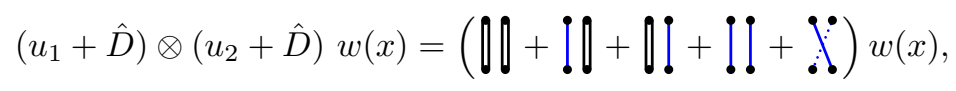

where \stands for $u_{1}$ or $u_{2}$, according to its position (for instance, $\|$ ! $=u_{1} \mathbb{I} \otimes$ $\frac{g x}{1-g x}$, and $\left.! \|=\frac{g x}{1-g x} \otimes \mathbb{I} u_{2}\right)$.

A.2. Co-derivatives of products. In this paper, we often use the co-derivatives acting on products, defining the quantities like $\hat{D}^{\otimes N} \Pi \cdot w(x)$ for an arbitrary class function $\Pi$. Then each co-derivative can act either on $\Pi$ or on $w(x)$, and at two spins, for instance, the Leibniz rule gives

$$
\begin{aligned}
& {[\hat{D} \otimes \hat{D} \quad \Pi w(x)]=\hat{D} \otimes\left(\left[\begin{array}{ll}
\hat{D} & \Pi
\end{array}\right] w(x)+\Pi[\hat{D} w(x)]\right)} \\
& =\left[\begin{array}{ll}
\hat{D} \otimes \hat{D} & \Pi
\end{array}\right] w(x)+\left[\begin{array}{ll}
\mathbb{I} \otimes \hat{D} & \Pi
\end{array}\right] \cdot\left[\begin{array}{ll}
\hat{D} \otimes \mathbb{I} & w(x)
\end{array}\right] \\
& +\left[\begin{array}{ll}
\hat{D} \otimes \mathbb{I} & \Pi
\end{array}\right] \cdot[\mathbb{I} \otimes \hat{D} w(x)]+\Pi\left[\begin{array}{ll}
\hat{D} \otimes \hat{D} & w(x)
\end{array}\right] \\
& =\nmid\left\{\begin{array}{l}
\Pi \\
w(x)
\end{array}+\underset{\downarrow}{\downarrow} \begin{array}{l}
\Pi \\
w(x)
\end{array}\right.
\end{aligned}
$$

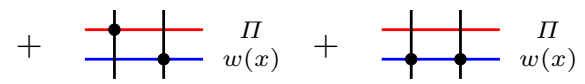

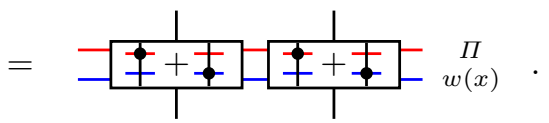

\footnotetext{
27 For the supergroups, $\mathcal{P}_{1,2}$ is replaced by the super permutation which differs from the usual permutation only by certain signs (see [1]).
} 
The equality between (66) and (67) is just the Leibniz rule, while (68) defines the graphical representation of each term of (67). Each black dot stands for a coderivative, acting on what lies on its right on the same horizontal line (horizontal lines actually hide auxiliary spaces whose characters contribute to $\Pi(g)$ resp $w(x)]$ ). The vertical lines correspond to the quantum space on which the whole operator acts, and the crossings without dots stand for $\mathbb{I}$. When operators are multiplied in the quantum spaces, they are represented one above another. For

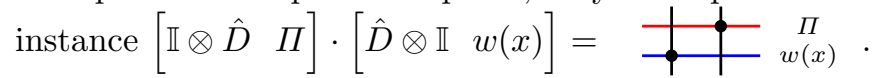

The last expression (69) gives a shorter representation of (67), where an implicit multiplication is taken between the blocks ++ . The generalization to an arbitrary number of spins $N$ is straightforward - one has $N$ such blocks instead of two.

One can also see that due to the relation $\hat{D} \operatorname{det}(g)=\mathbb{I} \operatorname{det}(g)$ and to the Leibniz rule, we have very generally

$$
\left[\bigotimes_{i}\left(u_{1}+2 \hat{D}\right) \Pi \operatorname{det}(g)^{a}\right]=\operatorname{det}(g)^{a}\left[\bigotimes_{i}\left(u_{1}+2 a+2 \hat{D}\right) \quad \Pi\right] .
$$

A.3. Commutativity of all T- and Q-operators. Everywhere through this paper, the quantities of interest are of the form $28\left[\bigotimes_{i=1}^{N}\left(u_{i}+\hat{D}\right) \Pi(g)\right]$, where $\Pi(g)$ is a class-invariant function of $g$ (a symmetric function of its eigenvalues).

In [1], the particular case when $\Pi$ is the character of a representation $\{\lambda\}$ was studied, and in particular it was proven that the T-operators commute for different representations $\{\lambda\}$ and $\{\mu\}$, yielding the relation

$$
\llbracket\left[\bigotimes_{i=1}^{N}\left(u_{i}+\hat{D}\right) \chi_{\{\lambda\}}(g)\right],\left[\bigotimes_{i=1}^{N}\left(u_{i}+v+\hat{D}\right) \chi_{\{\mu\}}(g)\right] \rrbracket=0
$$

where $\llbracket A, B \rrbracket \equiv A \cdot B-B \cdot A$, and $u_{j}, v \in \mathbb{C}$. Then, by writing an arbitrary class function as a linear combination of characters, one gets

$$
\llbracket\left[\bigotimes_{i=1}^{N}\left(u_{i}+\hat{D}\right) \Pi\right],\left[\bigotimes_{i=1}^{N}\left(u_{i}+v+\hat{D}\right) \Pi^{\prime}\right] \rrbracket=0,
$$

which holds when $\Pi, \Pi^{\prime}$ are two arbitrary class functions of $g$.

\section{B. Degree in $u$ of the polynomials $\mathbf{T}_{I}(u)$}

We already claimed that the l.h.s. of (12) only has simple poles. Now that we have described diagrammatic rules for the action of co-derivative on $w(z)$ functions, we can make this statement more explicit by writing the matrix coefficients of the Q-operator:

\footnotetext{
28 Most often, the operators of interest were actually of the form $\left[\bigotimes_{i=1}^{N}\left(u_{i}+2 \hat{D}\right) \Pi(g)\right]$, which is equivalent, after the rescaling $u_{i} \rightarrow 2 u_{i}$ and $\Pi \rightarrow \Pi / 2^{N}$, to $\left[\bigotimes_{i=1}^{N}\left(u_{i}+\hat{D}\right) \Pi(g)\right]$.
} 
These matrix elements are given by a generalization of the formula (4.16) [(35) in the arXiv version] of [1, which reads (see also (65) 29

$$
\begin{aligned}
{\left[\bigotimes_{i}\left(2+u_{i}+2 \hat{D}\right) w(t)\right]_{l_{1}, l_{2}, \cdots, l_{N}}^{k_{1}, k_{2}, \cdots, k_{N}} } & = \\
& =\sum_{\sigma \in \mathcal{S}_{N}} \prod_{i=1}^{N}\left(u_{i} \delta_{i, \sigma(i)}+\frac{2(g t)^{\theta(\sigma(i)-i-1)}}{1-g t}\right)_{l_{\sigma(i)}}^{k_{i}} w(t),
\end{aligned}
$$

where the sum is taken over the permutation group $\mathcal{S}_{N}$ of order $N$. At this point, we can already notice that the only non-zero terms in the sum are the permutations such that $\forall i, k_{i}=l_{\sigma(i)}$, since we are working in the diagonal basis. The same property holds for T-operators, and it implies for instance that the number of spins pointing in each direction $\left|e_{k}\right\rangle$ is a conserved quantity.

After multiplication by $(1-g t)^{\otimes N}$, the left-hand-side of (12) has only simple poles from $\left.w(z)\right|_{z \rightarrow 1 / x_{k}}$ :

$$
\begin{aligned}
& \left((1-g t)^{\otimes N} \cdot\left[\bigotimes_{i}\left(u_{i}+2 \hat{D}+2\right) w(t)\right]\right)_{l_{1}, l_{2}, \cdots l_{N}}^{k_{1}, k_{2}, \cdots k_{N}}= \\
& =\sum_{\sigma \in \mathcal{S}_{N}} \prod_{i=1}^{N} \delta_{l_{\sigma(i)}}^{k_{i}}\left(u_{i} \delta_{i, \sigma(i)}\left(1-x_{k_{i}} t\right)+2\left(x_{k_{i}} t\right)^{\theta(\sigma(i)-i-1)}\right) w(t)
\end{aligned}
$$

In the definition of $\mathbf{Q}_{\bar{j}}(u)$, we take the limit $t \rightarrow 1 / x_{j}$, and we see that $\mathbf{Q}_{\bar{\jmath}}(u)\left|e_{k_{1}, k_{2}, \cdots k_{N}}\right\rangle$ is independent of all $u_{n}$ such that $k_{n}=j$. As a consequence, the degree in $u$ of each eigenvalue of $\mathbf{Q}_{\bar{j}}(u)$ is equal to the number of spins pointing in the directions $\left|e_{k}\right\rangle_{k \neq j}$ in the corresponding eigenstate.

Moreover, the generalization to an arbitrary $\mathrm{T}$ or Q-operator is very simple: $\mathbf{T}_{I}^{\{\lambda\}}(u)\left|e_{l_{1}, l_{2}, \cdots, l_{N}}\right\rangle$ is independent of $u_{i}$ for all $i$ such that $l_{i} \in \bar{I}$, and the degree in $u$ of each eigenvalue of $\mathbf{T}_{I}^{\{\lambda\}}(u)$ is equal to the number of spins pointing in the directions $\left|e_{j}\right\rangle_{j \in I}$ in the corresponding eigenstate.

\section{Generating series of symmetric T-operators}

The TQ-relation (29) can be re-written as an expression for the generating series $30 \mathfrak{W}_{I}(u, z) \equiv \sum_{s=0}^{\infty} z^{s} \mathbf{T}_{I}^{s}(u)$ of symmetric T-operators: $\left.21,20,22,7\right]$

$$
\frac{\mathbf{Q}_{I, j}(u)}{\mathbf{Q}_{I}(u)} \mathfrak{W}_{I}(u, z)=\left(1-x_{j} \frac{\mathbf{Q}_{I}(u-2)}{\mathbf{Q}_{I}(u)} z e^{2 \partial_{u}}\right) \mathfrak{W}_{I, j}(u, z),
$$

where we used a shift operator $e^{2 \partial_{u}} f(u)=f(u+2)$ for any function $f(u)$. Let us then fix a chain of subsets of the full set: $\mathfrak{I}=I_{K} \supset I_{K-1} \supset \cdots \supset I_{0}=\emptyset$,

\footnotetext{
29 Here, the function $\theta(n)$ is equal to 1 [resp 0] if $n \geq 0$ [resp $n \leq-1]$.

30 From $\chi_{s}\left(g_{\emptyset}\right)=\delta_{s, 0}$, we can see that $\mathfrak{W}_{\emptyset}(u, z)=\mathbf{Q}_{\emptyset}(u)$, which we will use to get (75).
} 
where $I_{k}=\left\{j_{1}, j_{2}, \cdots, j_{k}\right\}, I_{k} \backslash I_{k-1}=\left\{j_{k}\right\}, k=1,2, \ldots, K$. Then from (74), we immediately get that

$$
\begin{aligned}
\mathfrak{W}_{I_{k}}(u, z) & =\mathcal{O}_{k} \cdot \mathcal{O}_{k-1} \cdots \mathcal{O}_{1} \cdot \mathbf{Q}_{\emptyset}(u) \\
\text { where } \mathcal{O}_{k} & =\left(1-x_{j_{k}} \frac{\mathbf{Q}_{I_{k-1}}(u-2)}{\mathbf{Q}_{I_{k-1}}(u)} z e^{2 \partial_{u}}\right)^{-1} \frac{\mathbf{Q}_{I_{k}}(u)}{\mathbf{Q}_{I_{k-1}}(u)} \\
& =\sum_{n=0}^{\infty}\left(x_{j_{k}} \frac{\mathbf{Q}_{I_{k-1}}(u-2)}{\mathbf{Q}_{I_{k-1}}(u)} z e^{2 \partial_{u}}\right)^{n} \frac{\mathbf{Q}_{I_{k}}(u)}{\mathbf{Q}_{I_{k-1}}(u)}
\end{aligned}
$$

which expresses the T-operators for symmetric tensor representations in terms of the Q-operators $\mathbf{Q}_{I_{n}}$. In particular, taking the coefficient of $z$ in (75), one gets the usual relation for the fundamental representation $s=1$ :

$$
\mathbf{T}_{I_{K}}^{1}(u)=\mathbf{Q}_{I_{K}}(u) \sum_{m=1}^{K} x_{j_{m}} \frac{\mathbf{Q}_{I_{m}}(u+2)}{\mathbf{Q}_{I_{m}}(u)} \frac{\mathbf{Q}_{I_{m-1}}(u-2)}{\mathbf{Q}_{I_{m-1}}(u)} .
$$

Note that this T-operator (76) has the same form as the T-function in 29 obtained by the nested Bethe ansatz, if the Q-operators are replaced by their eigenvalues (Q-functions).

The same generating series can also be written in the $g l(K \mid M)$ case for a fixed chain of subsets of the full set: $\mathfrak{I}=I_{K+M} \supset I_{K+M-1} \supset \cdots \supset I_{0}=\emptyset$, where $I_{k}=\left\{j_{1}, j_{2}, \cdots, j_{k}\right\}, I_{k} \backslash I_{k-1}=\left\{j_{k}\right\}, k=1,2, \ldots, K+M:$ (74) still holds for $j \leq K$, while for $\hat{l} \geq K+1$ (56) gives :

$$
\frac{\mathbf{Q}_{I}(u)}{\mathbf{Q}_{I, \hat{l}}(u)} \mathfrak{W}_{I, \hat{l}}(u, z)=\left(1-y_{l} \frac{\mathbf{Q}_{I, \hat{l}}(u-2)}{\mathbf{Q}_{I, \hat{l}}(u)} z e^{2 \partial_{u}}\right) \mathfrak{W}_{I}(u, z),
$$

so that (75) becomes for $g l(K \mid M)$ :

$$
\begin{array}{rlr}
\mathfrak{W}_{I_{k}}(u, z) & =\mathcal{O}_{k} \cdot \mathcal{O}_{k-1} \cdots \mathcal{O}_{1} \cdot \mathbf{Q}_{\emptyset}(u), \\
\text { where } \mathcal{O}_{k} & =\left(1-x_{j_{k}} \frac{\mathbf{Q}_{I_{k-1}}(u-2)}{\mathbf{Q}_{I_{k-1}}(u)} z e^{2 \partial_{u}}\right)^{-1} \frac{\mathbf{Q}_{I_{k}}(u)}{\mathbf{Q}_{I_{k-1}}(u)} \quad \text { if } j_{k} \leq K, \\
\mathcal{O}_{k} & =\frac{\mathbf{Q}_{I_{k}}(u)}{\mathbf{Q}_{I_{k-1}}(u)}\left(1-y_{l} \frac{\mathbf{Q}_{I_{k}}(u-2)}{\mathbf{Q}_{I_{k}}(u)} z e^{2 \partial_{u}}\right) & \text { if } j_{k}=\hat{l}>K
\end{array}
$$

which gives for instance the following generalization of (76) :

$$
\mathbf{T}_{I_{K+M}}^{1}(u)=\mathbf{Q}_{\bar{\emptyset}}(u) \sum_{k=1}^{K+M}(-1)^{p_{i_{k}}} \xi_{i_{k}} \frac{\mathbf{Q}_{I_{k-1}}\left(u-2(-1)^{p_{i_{k}}}\right) \mathbf{Q}_{I_{k}}\left(u+2(-1)^{p_{i_{k}}}\right)}{\mathbf{Q}_{I_{k-1}}(u) \mathbf{Q}_{I_{k}}(u)} .
$$

Note that the eigenvalue of (79) coincides with a traditional form of the Tfunction from the Bethe ansatz [38]. There are $(K+M)$ ! ways to chose the chain $\left\{I_{k}\right\}_{k=0}^{K+M}$, but (79) does not depend on this choice. This is the (super) Weyl group symmetry of the T-operators. 


\section{Bethe equations at the level of eigenvalues}

As explained in section 4.5, the operator equation (44) can be written as a Bethe equation (46) on the roots of the eigenvalues $\mathrm{Q}_{I}(u)$ of the polynomial operators $\mathbf{Q}_{I}(u)$ for any eigenstate.

The same can be easily done in the supersymmetric case so that (44), (61), (62) and (63) imply that for any eigenstate, the eigenvalues of the Q-operators satisfy the conditions 31 :

$$
\begin{aligned}
-1 & =\frac{x_{i}}{x_{j}} \frac{\mathrm{Q}_{I}\left(u_{k}^{(I, i)}-2\right) \mathrm{Q}_{I, i}\left(u_{k}^{(I, i)}+2\right) \mathrm{Q}_{I, i, j}\left(u_{k}^{(I, i)}\right)}{\mathrm{Q}_{I}\left(u^{(I, i)}\right) \mathrm{Q}_{I, i}\left(u_{k}^{(I, i)}-2\right) \mathrm{Q}_{I, i, j}\left(u_{k}^{(I, i)}+2\right)} & \text { for } 1 \leq k \leq K_{I, i}, \\
-1 & =\frac{y_{l}}{y_{m}} \frac{\mathrm{Q}_{I}\left(u_{k}^{(I, \hat{l})}+2\right) \mathrm{Q}_{I, \hat{l}}\left(u_{k}^{(I, \hat{l})}-2\right) \mathrm{Q}_{I, \hat{l}, \hat{m}}\left(u_{k}^{(I, \hat{l})}\right)}{\mathrm{Q}_{I}\left(u_{k}^{(I, \hat{l})}\right) \mathrm{Q}_{I, \hat{l}}\left(u_{k}^{(I, \hat{l})}+2\right) \mathrm{Q}_{I, \hat{l}, \hat{m}}\left(u_{k}^{(I, \hat{l})}-2\right)} & \text { for } 1 \leq k \leq K_{I, \hat{l}}, \\
1 & =\frac{x_{i}}{y_{l}} \frac{\mathrm{Q}_{I}\left(u_{k}^{(I, i)}-2\right) \mathrm{Q}_{I, i, \hat{l}}\left(u_{k}^{(I, i)}\right)}{\mathrm{Q}_{I}\left(u_{k}^{(I, i)}\right) \mathrm{Q}_{I, i, \hat{l}}\left(u_{k}^{(I, i)}-2\right)} & \text { for } 1 \leq k \leq K_{I, i}, \\
1 & =\frac{y_{l}}{x_{i}} \frac{\mathrm{Q}_{I}\left(u_{k}^{(I, \hat{l})}+2\right) \mathrm{Q}_{I, i, \hat{l}}\left(u_{k}^{(I, \hat{l})}\right)}{\mathrm{Q}_{I}\left(u_{k}^{(I, \hat{l})}\right) \mathrm{Q}_{I, i, \hat{l}}\left(u_{k}^{(I, \hat{l})}+2\right)} & \text { for } 1 \leq k \leq K_{I, \hat{l}},
\end{aligned}
$$

where $\left(u_{1}^{(I)}, \cdots, u_{K_{I}}^{(I)}\right)$ are the roots of the polynomial $\mathrm{Q}_{I}(u)$.

These equations (80,83) are equivalent to the traditional form of the Bethe equations [28, respectively, [cf. eqs. (68), (69), (71) and (70) in [22.]

\section{E. Proof of the master identity (8)}

In this appendix, we will give a proof of the master identity (8) based on the so-called Bazhanov-Reshetikhin formula 32 .

Consider the main object of our Master identity the generating operator of the transfer matrices

$$
\mathbf{W}_{I}(u)=\bigotimes_{i}\left(u_{i}+2 \hat{D}\right) \prod_{k \in I} w\left(z_{k}\right)
$$

where $z_{k} \in \mathbb{C}$ and $I$ is any subset of $\mathbb{Z}_{>0}$. We assume $z_{i} \neq z_{j}$ for any $i, j \in \mathbb{Z}_{>0}$ such that $i \neq j$. Then the master identity (8) can be rewritten in a form of a Plücker identity (or Jacobi identity):

$$
\left(z_{i}-z_{j}\right) \mathbf{W}_{I, i, j}(u+2) \mathbf{W}_{I}(u)=z_{i} \mathbf{W}_{I, j}(u) \mathbf{W}_{I, i}(u+2)-z_{j} \mathbf{W}_{I, j}(u+2) \mathbf{W}_{I, i}(u),
$$

\footnotetext{
31 Like in the section 5 we use here the convention $i, j \in\{1,2, \ldots, K\}$, and denote by $\hat{l}=l+K$ and $\hat{m}=m+K$ for $l, k \in\{1,2, \ldots, M\}$ for some indices of fermionic grading.

32 We thank Anton Zabrodin who proposed us the idea to use the Bazhanov-Reshetikhin formula for the proof of our master identity.
} 
where $i, j \in \mathbb{Z}_{>0}, i, j \notin I, i \neq j$. It can be solved recursively in the same way as the QQ-relations (40) giving

$$
\mathbf{W}_{I}(u)=\frac{\operatorname{det}\left(z_{j}^{n-k} \mathbf{W}_{j}(u-2 k+2)\right)_{1 \leq j, k \leq n}}{\operatorname{det}\left(z_{j}^{n-k}\right)_{1 \leq j, k \leq n} \prod_{k=1}^{n-1} \phi(u-2 k)} .
$$

Here $\phi(u) \equiv \mathbf{W}_{\emptyset}(u)=\prod_{j=1}^{N} u_{j}=\mathbf{Q}_{\bar{\emptyset}}(u)$, and we consider (without losing generality) the case $I=\{1,2, \ldots, n\}$ for any finite $n \in \mathbb{Z}_{>0}$.

Hence, to prove the master identity (8) all we need is to prove this determinant formula (86). Let us expand 33 the left-hand side of (86) multiplied by a factor with respect to $\left\{z_{k}\right\}$ :

$$
\begin{aligned}
& \mathbf{W}_{I}(u) \underset{1 \leq j, k \leq n}{\operatorname{det}}\left(z_{j}^{n-k}\right) \prod_{j=1}^{n} z_{j}^{j-n}=\operatorname{det}_{1 \leq j, k \leq n}\left(z_{j}^{j-k}\right) \mathbf{W}_{I}(u) \\
& =\bigotimes_{i}\left(u_{i}+2 \hat{D}\right) \sum_{\sigma \in S_{n}} \operatorname{sgn}(\sigma) \sum_{m_{1}=0}^{\infty} \sum_{m_{2}=0}^{\infty} \cdots \sum_{m_{n}=0}^{\infty} \prod_{k=1}^{n} \chi_{m_{k}}(g) z_{k}^{m_{k}-\sigma(k)+k},
\end{aligned}
$$

where the sum 34 is taken over the permutation group $S_{n}$ on the set $\{1,2, \ldots, n\}$ and $\operatorname{sgn}(\sigma)$ is a signature of the permutation $\sigma$. The coefficient of $\prod_{k=1}^{n} z_{k}^{\lambda_{k}}$ for any set of integers $\left\{\lambda_{k}\right\}_{k \in I}$ in (87), due to the Jacobi-Trudi formula (33), is nothing but the transfer matrix $T_{\lambda}$ from (6)

$$
\begin{aligned}
\bigotimes_{i}\left(u_{i}+2 \hat{D}\right) \sum_{\sigma \in S_{n}} \operatorname{sgn}(\sigma) \prod_{k=1}^{n} \chi_{\lambda_{k}+\sigma(k)-k}(g)= \\
=\bigotimes_{i}\left(u_{i}+2 \hat{D}\right) \operatorname{det}_{\substack{1 \leq j, k \leq n \\
n_{1}}}\left(\chi_{\lambda_{j}+k-j}(g)\right) .
\end{aligned}
$$

Now let us expand the right hand side of (866) (times the same $\left\{z_{k}\right\}$-dependent factor):

$$
\begin{aligned}
& \underset{1 \leq j, k \leq n}{\operatorname{det}}\left(z_{j}^{n-k} \mathbf{W}_{j}(u-2 k+2)\right) \prod_{j=1}^{n} z_{j}^{j-n} \\
& =\sum_{\sigma \in S_{n}} \operatorname{sgn}(\sigma) \sum_{m_{1}=0}^{\infty} \sum_{m_{2}=0}^{\infty} \cdots \sum_{m_{n}=0}^{\infty} \prod_{k=1}^{n} \mathbf{T}^{m_{k}}(u-2 \sigma(k)+2) z_{k}^{m_{k}-\sigma(k)+k} .
\end{aligned}
$$

The coefficient of $\prod_{k=1}^{n} z_{k}^{\lambda_{k}}$ in (89) is

$$
\sum_{\sigma \in S_{n}} \operatorname{sgn}(\sigma) \prod_{k=1}^{n} \mathbf{T}^{\lambda_{k}+\sigma(k)-k}(u-2 \sigma(k)+2)=\operatorname{det}_{1 \leq j, k \leq n}\left(\mathbf{T}^{\lambda_{j}+k-j}(u-2 k+2)\right) .
$$

33 Eq. (87) is an operator analogue of a generating function of the T-functions for any Young diagrams in eq. (2.44) in 23.

34 The sum over $\left\{m_{k}\right\}_{k \in I}$ can be taken over any integers since $\chi_{m}(g)=0$ if $m<0$. 
Therefore, the proof of (86) is reduced to the following identity

$$
\bigotimes_{i}\left(u_{i}+2 \hat{D}\right) \operatorname{det}_{1 \leq j, k \leq n}\left(\chi_{\lambda_{j}+k-j}(g)\right)=\frac{\operatorname{det}_{1 \leq j, k \leq n}\left(\mathbf{T}^{\lambda_{j}+k-j}(u-2 k+2)\right)}{\prod_{k=1}^{n-1} \phi(u-2 k)},
$$

where the l.h.s. is precisely the T-operator $\mathbf{T}^{\{\lambda\}}(u)$. We recognize here the Bazhanov-Reshetikhin formula proven in [1]. This proves the formulas (85)-(86), and hence the master identity (8) 35

\section{F. Co-derivative and "removal" of eigenvalues}

The co-derivative of $\chi_{s}\left(g_{I}\right)$ a priori does not have such a simple expression (as (64) in terms of diagrams) as the co-derivative of $\chi_{s}(g)$. We will see in this subsection how to define the action of co-derivative on $\chi_{s}\left(g_{I}\right)$, and then we will see how to compute the corresponding T-operators. In particular we will prove the relation (28).

\section{Definition.}

The action of co-derivatives on $w_{I}(z)$ (introduced in (23)), can be defined by means of equation (5), provided we specify what $x_{j}$ is at the point $e^{\phi \cdot e} g$. The most natural definition is based on the fact that $x_{j}$ is the $j^{\text {th }}$ eigenvalue of $g$, or in other words the $j^{\text {th }}$ root of its characteristic polynomial. In this sense, $x_{j}$ is a function of the group element $g: x_{j}=x_{j}(g)$. In particular, $x_{j}\left(\Omega g \Omega^{-1}\right)=x_{j}(g)$ for any similarity transformation.

If $g$ is a diagonal matrix, it is immediate to see that the contribution of the non-diagonal-elements of the matrix $e^{\phi \cdot e} g$ to the characteristic polynomial $\operatorname{det}\left(\lambda \mathbb{I}-e^{\phi \cdot e} g\right)$ is at least quadratic in $\phi$. This means that at the point $e^{\phi \cdot e} g$, $x_{j}$ is equal to $\left(e^{\phi \cdot e} g\right)_{j}^{j}$ to the first order in $\phi$. As a consequence, we get $\hat{D}_{j_{1}}^{i_{1}} x_{j}=$ $\hat{D}_{j_{1}}^{i_{1}} g_{j}^{j}=\delta_{j}^{i_{1}} \delta_{j_{1}}^{j} x_{j}$, so that $\hat{D} x_{j}=P_{j} x_{j}$, where the projector to the eigenspace for the $j$-th eigenvalue $x_{j}$ is $P_{j}=e_{j j}$ in this case.

More generally, if $g=\Omega^{-1} \tilde{g} \Omega$ where $\tilde{g}$ is diagonal and $\Omega$ is an arbitrary similarity transformation, ther 36 we obtain

$$
\begin{aligned}
\hat{D} x_{j} & =\left.\frac{\partial}{\partial \phi} x_{j}\left(e^{\phi \cdot e} \Omega^{-1} \tilde{g} \Omega\right)\right|_{\phi=0}=\left.\frac{\partial}{\partial \phi}\left(\Omega e^{\phi \cdot e} \Omega^{-1} \tilde{g}\right)_{j}^{j}\right|_{\phi=0} \\
& =\sum_{i_{1}, j_{1}} e_{i_{1} j_{1}} \Omega_{j_{1}}^{j}\left(\Omega^{-1}\right)_{j}^{i_{1}} x_{j}=\sum_{i_{1}, j_{1}} e_{i_{1} j_{1}}\left(\Omega^{-1} e_{j j} \Omega\right)_{j_{1}}^{i_{1}} x_{j}
\end{aligned}
$$

This exactly means that for a non-diagonal matrix $g, \hat{D} x_{j}=P_{j} x_{j}$, where the projector to the eigenspace for $x_{j}$ has the form $P_{j}=\Omega^{-1} e_{j j} \Omega$.

\footnotetext{
35 Note that both sides of (91) are antisymmetric w.r.t. the set $\left(\lambda_{1}-1, \lambda_{2}-2, \ldots, \lambda_{n}-n\right)$. Hence, when the determinant is non-zero, we can always relabel $\lambda_{k}$ 's in such a way that the highest weight components satisfy the usual inequalities: $\lambda_{1} \geq \lambda_{2} \geq \cdots \geq \lambda_{n} \geq 0$.

36 In (92), we mainly use the relation (2.6) [equation (12) in the arXiv version] of [1].
} 


\section{Computation of T-operators.}

The claim which was already given in (28) is that the computation of Toperators is done by commuting a factor $\frac{1}{w_{T}(z)}$ to the left of the co-derivatives.

We will show that in the definition (27) (or equivalently (26) ), the multiplication by $\mathbf{B}_{\bar{I}}$ introduced in (20) allows to commute any function of $x_{j}$ (where $j \in \bar{I}$ ) across the co-derivatives. As a consequence, the right hand sides of (27) and (28) are equal, giving

$$
\sum_{s=0}^{\infty} z^{s} \mathbf{T}_{I}^{s}(u)=\left(\prod_{j \in \bar{I}}\left(1-x_{j} z\right)\right) \lim _{\substack{t_{j} \rightarrow \frac{1}{x_{j}} \\ j \in \bar{I}}} \mathbf{B}_{\bar{I}} \cdot\left[\bigotimes_{i=1}^{N}\left(u_{i}+2 \hat{D}+2|\bar{I}|\right) w(z) \Pi_{\bar{I}}\right]
$$

where the r.h.s. can be easily computed by diagrammatic methods.

In the case of 1 spin, this is checked by computing 37

$$
\lim _{t \rightarrow \frac{1}{x_{j}}}(1-g t) \llbracket(u+2 \hat{D}), x_{j} \mathbb{I} \rrbracket=\left(1-\frac{g}{x_{j}}\right) \cdot\left(2 \hat{D} x_{j}\right)=2\left(1-\frac{g}{x_{j}}\right) x_{j} P_{j}=0
$$

We see that the key point in this commutation is the multiplication by $\lim _{t \rightarrow \frac{1}{x_{j}}}(1-$ $g t)=\left(1-g / x_{j}\right)$, which cancels the terms in $\hat{D} x_{j}=x_{j} P_{j}$ due to the property $\left(1-g / x_{j}\right) P_{j}=0$.

At $N=m+n$ spins $\left(m \in \mathbb{Z}_{\geq 0}, n \in \mathbb{Z}_{\geq 1}\right)$, the analogous relation is

$$
\begin{aligned}
& C_{m, n}=0, \quad \text { where } C_{m, n} \equiv\left(1-g / x_{j}\right)^{\otimes(m+n)} B_{m, n}, \\
& B_{m, n} \equiv\left(\bigotimes_{i=1}^{m}\left(u_{i}+2 \hat{D}\right)\right) \otimes \llbracket\left(u_{m+1}+2 \hat{D}\right), x_{j} \mathbb{I} \rrbracket \otimes\left(\bigotimes_{i=m+2}^{m+n}\left(u_{i}+2 \hat{D}\right)\right),
\end{aligned}
$$

and it is proven by the recurrence over $m$. For $m=0$, this follows from (94). Let's show how $C_{m+1, n}$ cancels under the assumption that $C_{m, n}=0$ for all $g \in G L(K)$ and any $u_{j} \in \mathbb{C}, j=1,2, \ldots, m+n$. Then for any $u_{0} \in \mathbb{C}$, one can calculate:

$$
\begin{aligned}
0 & =\left(\left(1-g / x_{j}\right) \otimes \mathbb{I}^{\otimes(m+n)}\right) \cdot\left(\left(u_{0}+2 \hat{D}\right) \otimes C_{m, n}\right) \\
& =C_{m+1, n}^{\prime}+2\left(\left(1-g / x_{j}\right) \otimes \mathbb{I}^{\otimes(m+n)}\right) \cdot\left[\hat{D} \otimes\left(1-g / x_{j}\right)^{\otimes(m+n)}\right] \cdot\left(\mathbb{I} \otimes B_{m, n}\right),
\end{aligned}
$$

37 Here, $\llbracket A, B \rrbracket$ denotes the commutator $A B-B A$. 
where 38

$$
\begin{aligned}
& C_{m+1, n}^{\prime} \equiv\left(1-g / x_{j}\right)^{\otimes(m+n+1)} B_{m+1, n}^{\prime}, \\
& B_{m+1, n}^{\prime} \equiv\left(\bigotimes_{i=0}^{m}\left(u_{i}+2 \hat{D}\right)\right) \otimes \llbracket\left(u_{m+1}+2 \hat{D}\right), x_{j} \mathbb{I} \rrbracket \otimes\left(\bigotimes_{i=m+2}^{m+n}\left(u_{i}+2 \hat{D}\right)\right),
\end{aligned}
$$

and due $\operatorname{td} 39 \hat{D} \otimes g / x_{j}=\mathcal{P} \cdot\left(1 \otimes g / x_{j}\right)-P_{j} \otimes g / x_{j}$, the second term in (96) (multiplied by $1 / 2$ ) can be expanded to get

$$
\begin{aligned}
& -\frac{1}{2} C_{m+1, n}^{\prime}= \\
= & \left(\left(1-g / x_{j}\right) \otimes \mathbb{I}^{\otimes(m+n)}\right) \cdot\left(\sum_{k=1}^{m+n} \mathcal{P}_{0 k} \cdot \mathbb{I} \otimes\left(\bigotimes_{i=1}^{m+n}\left(1-\delta_{i}^{k}-g / x_{j}\right)\right)\right) \cdot\left(\mathbb{I} \otimes B_{m, n}\right) \\
- & \left(\left(1-g / x_{j}\right) \otimes \mathbb{I}^{\otimes(m+n)}\right) \cdot\left(\sum_{k=1}^{m+n} P_{j} \otimes\left(\bigotimes_{i=1}^{m+n}\left(1-\delta_{i}^{k}-g / x_{j}\right)\right)\right) \cdot\left(\mathbb{I} \otimes B_{m, n}\right),
\end{aligned}
$$

and the first term becomes

$$
-\sum_{k} \mathcal{P}_{0 k} \cdot\left(\mathbb{I}^{\otimes k} \otimes\left(g / x_{k}\right) \otimes \mathbb{I}^{\otimes(n+m-k)}\right) \cdot\left(\mathbb{I} \otimes\left(1-g / x_{k}\right)^{\otimes(m+n)}\right) \cdot\left(\mathbb{I} \otimes B_{m, n}\right),
$$

which 40 is zero because it contains $C_{m, n}$. The second term is also zero because it contains $\left(1-g / x_{j}\right) P_{j}$. This completes the proof of the fact that $C_{m+1, n}^{\prime}=0$, from which $C_{m+1, n}=0$ follows.

As a consequence, we can indeed commute the factors $\frac{1}{w_{\bar{I}}(z)}$ to the left of all co-derivatives in (27) and get the relation (28).

38 In (96), we used the Leibniz rule $\hat{D} \otimes\left(\left(1-g / x_{j}\right)^{\otimes m+n} \cdot B_{m, n}\right)=\left[\hat{D} \otimes\left(1-g / x_{j}\right)^{\otimes m+n}\right]$. $\left(\mathbb{I} \otimes B_{m, n}\right)+\left(\mathbb{I} \otimes\left(1-g / x_{j}\right)^{\otimes m+n}\right) \cdot\left[\hat{D} \otimes B_{m, n}\right]$.

39 Here, the projector $P_{j}$ on the the $j^{\text {th }}$ should not be confused with the permutation operator $\mathcal{P}$ between the quantum spaces.

40 Here, we use the fact that $\left(\left(1-\frac{g}{x_{j}}\right) \otimes \mathbb{I}^{\otimes(m+n)}\right) \cdot \mathcal{P}_{0, k}=\mathcal{P}_{0, k} \cdot \mathbb{I}^{\otimes k} \otimes\left(1-g / x_{j}\right) \otimes \mathbb{I}^{\otimes n+m-k}$. 


\section{References}

1. V. Kazakov and P. Vieira, "From Characters to Quantum (Super)Spin Chains via Fusion," JHEP 0810 (2008) 050 [arXiv:0711.2470 [hep-th]].

2. V. Bazhanov and N. Reshetikhin, "Restricted Solid On Solid Models Connected With Simply laced Algebras And Conformal Field Theory," J. Phys. A 23, 1477 (1990).

3. A. Klümper and P. A. Pearce, "Conformal weights of RSOS lattice models and their fusion hierarchies" Physica A 183 (1992) 304.

4. A. Kuniba, T. Nakanishi and J. Suzuki, "Functional relations in solvable lattice models. 1: Functional relations and representation theory," Int. J. Mod. Phys. A 9 (1994) 5215 [arXiv:hep-th/9309137].

5. A. Kuniba, S. Nakamura and R. Hirota, J. Phys. A 29, 1759 (1996) [arXiv:hepth/9509039].

6. V. V. Bazhanov, S. L. Lukyanov and A. B. Zamolodchikov, "Integrable Structure of Conformal Field Theory II. Q-operator and DDV equation," Commun. Math. Phys. 190 (1997) 247 [arXiv:hep-th/9604044].

7. Z. Tsuboi, "Analytic Bethe ansatz and functional equations for Lie superalgebra sl $(r+$ $1 \mid s+1)$," J. Phys. A 30, 7975 (1997) [arXiv:0911.5386 [math-ph]].

8. N. Gromov, V. Kazakov and P. Vieira, "Finite Volume Spectrum of 2D Field Theories from Hirota Dynamics," JHEP 0912, 060 (2009) [arXiv:0812.5091 [hep-th]].

9. V. Kazakov and S. Leurent, "Finite Size Spectrum of SU(N) Principal Chiral Field from Discrete Hirota Dynamics," arXiv:1007.1770 [hep-th].

10. N. Gromov, V. Kazakov and P. Vieira, "Exact Spectrum of Anomalous Dimensions of Planar N=4 Supersymmetric Yang-Mills Theory," Phys. Rev. Lett. 103 (2009) 131601 [arXiv:0901.3753 [hep-th]].

11. D. Bombardelli, D. Fioravanti and R. Tateo, "Thermodynamic Bethe Ansatz for planar AdS/CFT: a proposal," J. Phys. A 42, 375401 (2009) [arXiv:0902.3930 [hep-th]]. ^ N. Gromov, V. Kazakov, A. Kozak and P. Vieira, "Integrability for the Full Spectrum of Planar AdS/CFT II," Lett. Math. Phys. 91, 265 (2010) [arXiv:0902.4458 [hep-th]]. ^ G. Arutyunov and S. Frolov, "Thermodynamic Bethe Ansatz for the AdS $S_{5} \times S^{5}$ Mirror Model," JHEP 0905, 068 (2009) [arXiv:0903.0141 [hep-th]].

12. I. Cherednik, On special basis of irreducible representations of degenerated affine Hecke algebras, Funk. Analys. i ego Prilozh. 20:1 (1986) 87-88 (in Russian) ^ I. Cherednik, Quantum groups as hidden symmetries of classical representation theory, Proceed. of 17th Int. Conf. on diff. geom. methods in theoretical physics, World Scient. (1989), 47. $\star$ I. Cherednik, On irreducible representations of elliptic quantum $R$-algebras, Dokl. Akad. Nauk SSSR 291:1, 49-53 (1986) Translation: M 34-1987, 446-450. ^ I. Cherednik, An analogue of character formula for Hecke algebras, Funct. Anal. and Appl. 21:2, 94-95 (1987) (translation: pgs 172-174).

13. R.J. Baxter, Partition function of the eight-vertex lattice model, Ann. Phys. 70 (1972) 193-228.

14. V. V. Bazhanov, S. L. Lukyanov and A. B. Zamolodchikov, "Integrable structure of conformal field theory. III: The Yang-Baxter relation," Commun. Math. Phys. 200, 297 (1999) [arXiv:hep-th/9805008].

15. V. Pasquier, M. Gaudin, The periodic Toda chain and a matrix generalization of the Bessel function recursion relations, J. Phys. A: Math. Gen. 25 (1992) 5243-4252; ^ K. Hikami, Baxter Equation for Quantum Discrete Boussinesq Equation, Nucl. Phys. B604 (2001) 580-602 [arXiv:nlin/0102021]; $\star$ K. Fabricius, B.M. McCoy, New Developments in the Eight Vertex Model, J.Statist.Phys. 111 (2003) 323-337 [arXiv:cond-mat/0207177]; ^ V.B. Kuznetsov, V.V. Mangazeev, E.K. Sklyanin, Q-operator and factorised separation chain for Jack polynomials, Indag. Math. 14 (2003) 451-482 [arXiv:math/0306242[math.CA]]; $\star$ P. P. Kulish, A. M. Zeitlin, "Superconformal field theory and SUSY N=1 KDV hierarchy II: The Q-operator," Nucl. Phys. B709 (2005) 578 [hep-th/0501019]; ^ C. Korff, A Q-Operator Identity for the Correlation Functions of the Infinite XXZ SpinChain, J.Phys. A: Math. Gen. 38 (2005) 6641-6658 [arXiv:hep-th/0503130]; ^ A.G. Bytsko, J. Teschner, Quantization of models with non-compact quantum group symmetry. Modular XXZ magnet and lattice sinh-Gordon model, J.Phys.A 39 (2006) 1292712981[arXiv:hep-th/0602093]; ^ H. Boos, M. Jimbo, T. Miwa, F. Smirnov, Y. Takeyama, Hidden Grassmann structure in the XXZ model, Commun. Math. Phys. 272 (2007) 263281 [arXiv:hep-th/0606280]; ^ T. Kojima, "The Baxter's Q-operator for the W-algebra $W_{N}$," J.Phys.A: Math. Theor. 41 (2008) 355206 [arXiv:0803.3505 [nlin.SI]]. ^ S.E. Derkachov, A.N. Manashov, Factorization of R-matrix and Baxter Q-operators for generic $s l(N)$ 
spin chains, J.Phys.A: Math. Theor. 42 (2009) 075204; [arXiv:0809.2050 [nlin.SI]]. \ H. Boos, F. Göhmann, A. Klümper, K.S. Nirov, A.V. Razumov, Exercises with the universal R-matrix J. Phys. A: Math. Theor. 43 (2010) 415208 [arXiv:1004.5342 [math-ph]]. $\star$ V. V. Bazhanov, T. Lukowski, C. Meneghelli and M. Staudacher, "A Shortcut to the Q-Operator,” J. Stat. Mech. 1011 (2010) P11002 [arXiv:1005.3261 [hep-th]]. ^ S.E. Derkachov, A.N. Manashov, "Noncompact sl $(N)$ spin chains: Alternating sum representation for finite dimensional transfer matrices," arXiv:1008.4734 [nlin.SI].

16. V. V. Bazhanov, A. N. Hibberd and S. M. Khoroshkin, "Integrable structure of $W_{3}$ conformal field theory, quantum Boussinesq theory and boundary affine Toda theory," Nucl. Phys. B 622 (2002) 475 [arXiv:hep-th/0105177].

17. A. V. Belitsky, S. E. Derkachov, G. P. Korchemsky and A. N. Manashov, "Baxter Qoperator for graded $S L(2 \mid 1)$ spin chain," J. Stat. Mech. 0701, P005 (2007) [arXiv:hepth/0610332].

18. V. V. Bazhanov and Z. Tsuboi, "Baxter's Q-operators for supersymmetric spin chains," Nucl. Phys. B 805, 451 (2008) [arXiv:0805.4274 [hep-th]]; in section 2.4 of this paper, solutions of the Yang-Baxter relation (L-operators) for the Q-operators in the $U_{q}(\widehat{s l}(2 \mid 1))$ case were presented and the Q-operators were given as the (super)trace of these L-operators over some oscillator representations. An idea of the derivation of these solutions of the Yang-Baxter relation for the Q-operators was presented at the "Workshop and Summer School: From Statistical Mechanics to Conformal and Quantum Field Theory", the university of Melbourne, January, 2007 and La 79eme Rencontre entre physiciens theoriciens et mathematiciens "Supersymmetry and Integrability", IRMA Strasbourg, June, 2007.

19. V. V. Bazhanov, R. Frassek, T. Lukowski, C. Meneghelli and M. Staudacher, "Baxter Q-Operators and Representations of Yangians ," arXiv:1010.3699 [math-ph]. The results of this paper have been presented as a talk of M.Staudacher at a conference "Integrability in Gauge and String Theory 2010" (Nordita, Sweden, 28 June 2010).

20. V. Kazakov, A. S. Sorin and A. Zabrodin, "Supersymmetric Bethe ansatz and Baxter equations from discrete Hirota dynamics," Nucl. Phys. B 790, 345 (2008) [arXiv:hepth/0703147].

21. I. Krichever, O. Lipan, P. Wiegmann and A. Zabrodin, "Quantum integrable models and discrete classical Hirota equations,” Commun. Math. Phys. 188 (1997) 267 [arXiv:hepth/9604080].

22. A. Zabrodin, "Backlund transformations for difference Hirota equation and supersymmetric Bethe ansatz," Theor. Math. Phys. 155, 567 (2008) [arXiv:0705.4006 [hep-th]].

23. Z. Tsuboi, "Solutions of the T-system and Baxter equations for supersymmetric spin chains," Nucl. Phys. B 826 (2010) 399 [arXiv:0906.2039 [math-ph]].

24. G.P. Pronko, Yu.G. Stroganov, "Families of solutions of the nested Bethe Ansatz for the $A_{2}$ spin chain," J. Phys. A: Math. Gen. 33 (2000) 8267-8273 [arXiv:hep-th/9902085]; ^ P. Dorey, C. Dunning, D. Masoero, J. Suzuki, R. Tateo, "Pseudo-differential equations, and the Bethe Ansatz for the classical Lie algebras," Nucl. Phys. B 772 (2007) 249-289 [arXiv:hep-th/0612298].

25. F. Göhmann, A. Seel: "A note on the Bethe Ansatz solution of the supersymmetric $t-J$ model," Czech.J.Phys. 53 (2003) 1041 [arXiv:cond-mat/0309138].

26. N. Gromov and P. Vieira, "Complete 1-loop test of AdS/CFT," JHEP 0804, 046 (2008) [arXiv:0709.3487 [hep-th]].

27. F. Woynarovich, "Low-energy excited states in a Hubbard chain with on-site attraction," J.Phys.C: Solid State Phys. 16 (1983) 6593; ^ P.A. Bares, I.M.P. Carmelo, J. Ferrer, P. Horsch, "Charge-spin recombination in the one-dimensional supersymmetric t-J model," Phys. Rev. B46 (1992) 14624. ^ Z. Tsuboi, "Analytic Bethe Ansatz and functional equations associated with any simple root systems of the Lie superalgebra $\operatorname{sl}(r+1 \mid s+1)$ ", Physica A 252, 565 (1998) [arXiv:0911.5387 [math-ph]].

28. C.K. Lai, "Lattice gas with nearest neighbor interaction in one dimension with arbitrary statistics," J. Math. Phys. 15 (1974) 1675. ^ B. Sutherland, "Model for a multicomponent quantum system," Phys. Rev. B12 (1975) 3795.

29. O. Babelon, H.J. de Vega, C-M. Viallet, "Exact solution of the $Z_{n+1} \times Z_{n+1}$ symmetric generalization of the XXZ model," Nucl. Phys. B200 (1982) 266. ^ P. P. Kulish, N. Y. .Reshetikhin, "Diagonalization Of $G l(n)$ Invariant Transfer Matrices and Quantum N Wave System (Lee Model),” J. Phys. A A16 (1983) L591.

30. T. Deguchi, P.P. Martin, "An Algebraic Approach to Vertex Models and Transfer-Matrix Spectra, ” Int. J. Mod. Phys. A7, Suppl. 1A (1992) 165.

31. N. Gromov, V. Kazakov, S. Leurent and Z. Tsuboi, "Wronskian Solution for AdS/CFT Y-system," JHEP 1101 (2011) 155 [arXiv:1010.2720 [hep-th]]. 
32. L. D. Faddeev, arXiv:hep-th/9605187. ^ Andreas Klümper, Integrability of quantum chains: theory and applications to the spin-1/2 XXZ chain, arXiv:cond-mat/0502431.

33. N. Gromov, V. Kazakov and Z. Tsuboi, "PSU $(2,2 \mid 4)$ Character of Quasiclassical AdS/CFT," JHEP1007(2010)097 [arXiv:1002.3981 [hep-th]].

34. A. Hegedus, "Discrete Hirota dynamics for AdS/CFT," Nucl. Phys. B 825, 341 (2010) [arXiv:0906.2546 [hep-th]].

35. V. V. Bazhanov, S. L. Lukyanov and A. B. Zamolodchikov, "Quantum field theories in finite volume: Excited state energies," Nucl. Phys. B 489 (1997) 487 [arXiv:hepth/9607099].

36. P. Dorey and R. Tateo, "Excited states by analytic continuation of TBA equations," Nucl. Phys. B 482, 639 (1996) [arXiv:hep-th/9607167].

37. N. Gromov, V. Kazakov and P. Vieira, "Exact AdS/CFT spectrum: Konishi dimension at any coupling," Phys. Rev. Lett. 104, 211601 (2010) [arXiv:0906.4240 [hep-th]].

38. C.L. Schultz, "Eigenvectors of the multicomponent generalization of the six-vertex model," Physica A122 (1983) 71. 